\title{
Neutral and Cationic Palladium Complexes of P-Stereogenic Phosphanes Bearing a Heterocyclic Substituent
}

(1)

[a] Departament de Química Inorgànica i Orgànica, Secció de Química Inorgànica, Universitat de Barcelona, Martí i Franquès, 1-11, 08028 Barcelona, Spain

E-mail: arnald.grabulosa@qui.ub.es

http://www.ub.edu/inorgani/ca/index.html

[b] Departament de Cristallografia, Mineralogia i Dipòsits Minerals, Universitat de Barcelona, Martí i Franquès, s/n, 08028 Barcelona, Spain

E-mail: arnald.grabulosa@qi.ub.es 
41 ABSTRACT:

42

43 The coordination chemistry of 13 optically pure Pstereogenic diarylmonophosphanes $\mathrm{P}(\mathrm{Het}) \mathrm{PhR}[\mathrm{Het}=$ 44 4-dibenzofuranyl (DBF), 4-dibenzothiophenyl (DBT), 4-dibenzothiophenyl S,S-dioxide (DBTO2) and

45 1-thianthrenyl (TA); R = OMe, Me, iPr, Fc (ferrocenyl)] to Pd-allyl moieties is described. Both neutral

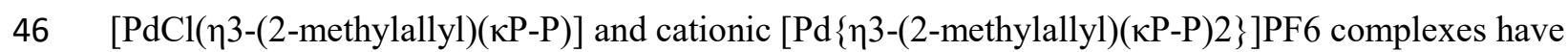

47 been prepared. Coordination of the heteroatom of the heterocycle was only possible in the case of TA-

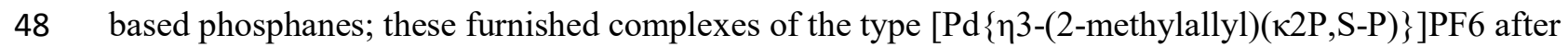

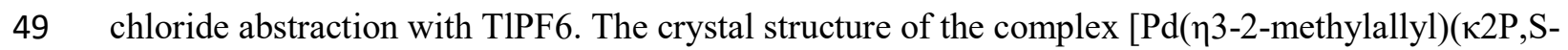
$50 \mathrm{PPh}(\mathrm{OMe})(1-\mathrm{TA})] \mathrm{PF} 6$ is reported. The neutral $\mathrm{Pd}$ complexes were found to be highly active in the 51 hydrovinylation of styrene after activation with AgBF4, except for the TAbased phosphanes. The 52 cationic Pd complexes were evaluated in allylic alkylation and amination with the model substrate 53 ractrans-1,3-diphenylprop-2-enyl acetate (rac-I), achieving total conversions and up to $70 \%$ ee. 


\section{INTRODUCTION}

59 Although chiral diphosphanes are the most successful type of ligands of transition-metal homogeneous

60 catalysis, for certain reactions or under certain conditions monophosphorus ligands can give better

61 results or they can even be required, due to mechanistic restrictions. One example is the Ni- or Pd-

62 catalysed hydrovinylation of activated olefins.[1]

63 In these processes, it is thought that secondary (hemilabile) interactions can play a crucial role,

64 improving the activity and selectivity of the reaction. Several elegant examples have been provided by

65 RajanBabu and co-workers[2] and by Franciò, Leitner and co-workers,[3] who convincingly

66 demonstrated the importance of secondary interactions in Ni-catalysed hydrovinylation of olefins. The

67 design of the ligand, however, is not easy, because it has to contain the appropriate Lewis base suitably

68 located in the scaffold of the ligand to interact with the metal centre during the catalytic reaction.

69 Very recently,[4] we described a series of monophosphorus, P-stereogenic ligands containing a

70 heterocyclic substituent [4-dibenzofuryl (DBF), 4-dibenzothiophenyl (DBT), 4-dibenzothiophenyl

71 (DBTO2) and 1-thianthrenyl (TA)] designed with the aim of disposing the heteroatom of the heterocycle

72 in a suitable position allowing it to interact with the metal atom (Scheme 1).

73 The coordination chemistry towards $\mathrm{Ru}-\eta 6$-arene moieties and the application of the complexes to

74 transfer hydrogenation was also described. It was found that the sulfur atoms of DBTand TA-containing

75 ligands were able to act in conjunction with phosphorus as bidentate ligands in a $\kappa 2 \mathrm{P}, \mathrm{S}$-coordinated

76 fashion.

77 In this paper we describe the coordination of these ligands to Pd- $\eta 3$-allylic moieties and the application

78 of the obtained complexes to catalytic hydrovinylation and allylic substitution reactions. 


\section{Neutral Complexes}

83 As previously described for other monophosphanes,[5] treatment of the well-known Pd-dimer D with slightly more than two equivalents of phosphane in dichloromethane yielded the expected neutral complexes Pd1-13, of the type $[\mathrm{PdCl}(\eta 3-2-$ methylallyl)(P)] (Scheme 2).

The complexes were obtained as pale yellow solids, except for those containing phosphanes bearing the ferrocenyl group (Pd8 and Pd12), which were red. The complexes were characterised by IR, chemical microanalysis (or MS) and multinuclear NMR in solution. As expected, $[5,6]$ the complexes were found to exist as mixtures of two diastereomeric species in solution, due to the presence of the chiral ligand and the allyl moiety. Hence, two singlets in the $31 \mathrm{P}\{1 \mathrm{H}\}$ NMR spectra, often partially overlapped, could be observed. All of the $\mathrm{C}$ and $\mathrm{H}$ atoms of the complexes are in principle different in each diastereomer; this could be clearly seen in the duplication of signals in the part corresponding to the allyl moiety in the $1 \mathrm{H}$ and 13C NMR spectra of the complexes. Full details can be found in the Experimental Section. Integration of the $31 \mathrm{P}$ and $1 \mathrm{H}$ NMR spectra allows the estimation of the diastereomeric ratio in solution. It was found that this was approximately 1:1 for all complexes, except for Pd12, for which it was 1:1.2. Interestingly, in complexes bearing a phosphane containing the thianthryl group ( $\mathrm{Pd} 3$ and $\mathrm{Pd} 13)$ the $\mathrm{H}$ atoms of the allyl group gave rise to extremely wide peaks in the 1H NMR spectra. In addition, no peaks could be detected for the allyl group in the $13 \mathrm{C}\{1 \mathrm{H}\}$ spectra at room temperature. Low-temperature $1 \mathrm{H}$ NMR spectra of Pd13 in CD2Cl2 (see the Supporting Information) showed, however, that the expected allylic hydrogen atoms appeared when the spectrum was recorded at $-80{ }^{\circ} \mathrm{C}$.

\section{Cationic Complexes}

103 The next type of Pd complexes prepared were cationic bisphosphanes of the type $[\operatorname{Pd}(\eta 3-2-$ methylallyl)(P)2]PF6. As described in previous reports,[5,7] they were obtained by splitting dimer D with slightly more than four equivalents of phosphane in the presence of an excess of ammonium hexafluorophosphate (Scheme 3).

The complexes were obtained as stable brown solids after extractive workup with water to remove inorganic salts. They were characterised by the usual techniques. The NMR spectra showed that a single species was present in solution, as previously found for analogous compounds.[5,7] The presence of the allyl group and the chirality of the phosphane makes it the case that the atoms in the molecule are all different. Therefore, two sharp doublets in the $31 \mathrm{P}\{1 \mathrm{H}\}$ NMR spectra corresponding to the two coupled phosphorus atoms $(2 \mathrm{JP}, \mathrm{P}=30-57 \mathrm{~Hz})$ could be observed. In the 13C NMR spectra the two terminal allyl carbon atoms appeared as doublets or doublets of doublets, due to the coupling with the $\mathrm{P}$ atoms of the phosphanes. The differences between the $13 \mathrm{C}$ chemical shifts of these two atoms are small $(<2$ ppm), as found in similar complexes.[7] In the 1H NMR spectra, the four resonances of the allylic $\mathrm{H}$ atoms appeared as two broad singlets, corresponding to the syn protons and two doublets, corresponding 
to the anti protons $\left(2 \mathrm{JH}, \mathrm{P}=9-12 \mathrm{~Hz}\right.$ ). The preparation of $\mathrm{Pd} 44^{\prime}$ in pure form was not possible because it was always contaminated with around $25 \%$ of neutral Pd4.

119 We next moved to study of the coordinative interactions between the heterocycle and the Pd centre. Following our previous report with ruthenium,[4] we treated the neutral complexes Pd7 and Pd10 with thallium hexafluorophosphate in dichloromethane, and the solid obtained after the filtration of $\mathrm{TlCl}$ and removal of the solvent was analysed by NMR (Scheme 4). In the case of Pd7, no peaks appeared in the 31P NMR spectrum and the 1H NMR spectrum was broad and uninformative, indicating that no definite species were formed. In the case of the solid obtained from Pd10, both 31P and 1H NMR spectra showed that it corresponded to bis(phosphane) complex $\operatorname{Pd} 10^{\prime}$. The formation of this compound indicates that a symmetrisation (disproportionation) reaction yielding the bis(phosphane) and the bis(solvato) complexes had taken place, as previously reported for Pd complexes with other monophosphane ligands.[7,8]

In contrast to the unsuccessful attempts described above, the coordination of the $\mathrm{S}$ atom of the thianthryl group in complexes Pd3 and Pd13 was successfully accomplished, yielding cationic complexes Pd3" and Pd13" as pale yellow solids after recrystallisation (Scheme 5). It should be mentioned that the NMR of Pd13" shows the presence of a small quantity of bis(phosphane) complex Pd13'.

The $\kappa 2 \mathrm{P}, \mathrm{S}$ complexation of the ligands was confirmed by the downfield shift of the 31P signals $\left[\Delta \delta\left(\operatorname{Pd} 3^{\prime \prime}-\mathrm{Pd} 3\right)=26.2 \mathrm{ppm} ; \Delta \delta\left(\operatorname{Pd} 13^{\prime \prime}-\mathrm{Pd} 13\right)=26.4 \mathrm{ppm}\right]$ characteristic when a five-membered ring is formed.[9] Two peaks appeared in the $31 \mathrm{P}\{1 \mathrm{H}\}$ spectra and two sets of signals were present in the $1 \mathrm{H}$ and $13 \mathrm{C}\{1 \mathrm{H}\}$ spectra, indicating that complexes Pd3" and Pd13" exist as mixtures of the two diastereomers, as was also the case with precursor complexes Pd3 and Pd13. The ratio between the cationic complexes is roughly $60: 40$, meaning that the sulfur complexation step occurs with a small degree of diastereoselectivity. It is worth noting that complexes Pd3" and Pd13" showed well-defined NMR spectra. In the $1 \mathrm{H}$ and $13 \mathrm{C}\{1 \mathrm{H}\}$ NMR spectra, the expected two sets of peaks assignable to the allyl group appear, in contrast with the cases of Pd3 and Pd13 (vide infra). This is probably due to the rigid $\kappa 2 \mathrm{P}, \mathrm{S}$-coordination of the ligand in the cationic complexes.

For complex Pd3", single crystals suitable for X-ray crystallography could be obtained. A representation of its molecular structure is given in Figure 1.

The unit cell of Pd3" contains two independent molecules, corresponding to the two different isomers of the complex. They can be named as syn and anti with regard to the relative disposition between the methyl group of the allyl fragment and the methoxy group of the phosphinite. A particular feature of the structure is the expected[10] nonplanar, "butterfly" shape of the thianthryl substituent of the phosphane, which is folded along its $\mathrm{S}-\mathrm{S}$ axis. Interestingly, for both isomers in the crystal the thianthrene moiety is folded such that it remains parallel to the $\mathrm{C}(20)-\mathrm{C}(22)$ bond of the allyl group. The Pd atom is in a distorted square-planar environment, the interatomic distances and angles of which are similar in the two isomers present in the unit cell and also similar to those in other Pd complexes containing fivemembered $\mathrm{P}, \mathrm{S}$ chelate rings, such as in a complex with a 4-diphenylphosphinophenothiazine ligand 
described recently by Silaghi-Dumitrescu and co-workers.[11] The distance between the Pd atom and the $\mathrm{C}$ atom of the allyl group trans to the $\mathrm{P}$ atom is larger than with the $\mathrm{C}$ atom trans to the $\mathrm{S}$ atom, indicating a higher trans influence of the phosphinite group relative to the thioether group. It should be pointed out that the $\mathrm{S}$ atom coordinated to $\mathrm{Pd}$ is a stereogenic centre and that each of the molecules in the crystal structure has a different absolute configuration.

The complexation studies described here with the Pd- $\eta 3$-methallyl moiety can be compared with analogous recently described studies with the $\mathrm{Ru}-\eta 6$-arene moiety.[4] For the Ru systems it was found that both DBT- and TA-based phosphanes effectively coordinated to the Ru atom in a $22 \mathrm{P}, \mathrm{S}$-mode but the DBF-based ligands did not. This shows that with the systems studied, the DBF-based phosphanes have the weakest tendency to act as bidentate ligands, whereas the TA-based ones show the strongest tendency. The softer character of sulfur relative to oxygen and the greater flexibility of the TA group relative to DBF and DBT probably account for the differences in coordination abilities of these ligands.

\section{Pd-Catalysed Hydrovinylation}

The hydrovinylation reaction is a catalysed heterocodimerisation between ethylene and a conjugated diene. $[1 \mathrm{~b}, 1 \mathrm{~d}]$ This reaction is interesting because it creates a $\mathrm{C}-\mathrm{C}$ bond by using ethylene, which is an inexpensive feedstock, and because the double bond incorporated into the molecule can subsequently be manipulated in a multitude of ways. In addition, because a stereogenic centre is created, the reaction can be carried out enantioselectively.[1a,1c,1d] Despite its interest, activity and selectivity issues hamper its full development. The most typical catalytic systems involve a NiII or PdII precursor stabilised with a monophosphorus ligand because for these metals bidentate ligands inhibit the reaction.[2c] The presence of groups capable of establishing secondary coordination interactions with the metal can be beneficial for the reaction, as shown by RajanBabu and co-workers[2b,12] and by Leiner and co-workers[3a,13] in Ni-based systems. The catalytically active species is thought to be a metal hydride. In general, nickel systems are more commonly used because they are very active and can be highly enantioselective but with the penalty of requiring (usually)[3b] very low temperatures. In contrast, palladium-based systems work well at room temperature. $[6,14]$ The challenge, apart from improving the enantioselectivity, is the control of the regioselectivity of the reaction, because the same hydrovinylation catalyst tends to isomerise the initially formed 3-arylbut-2-enes to the more stable 2-arylbut-2-enes. It has been found by us $[5,6,8 b, 14 b, 15]$ and by others[14a] that $[\mathrm{PdCl}(\eta 3$-allyl)P] $(\mathrm{P}=$ monophosphorus ligand $)$ complexes are excellent catalytic precursors of the active species after halide abstraction, so the potential of complexes Pd1-13 in hydrovinylation was explored (Scheme 6).

The activation of the catalysts was carried out with silver tetrafluoroborate in the presence of styrene. After removal of silver chloride by filtration, the solution was pressurised with ethylene at $25^{\circ} \mathrm{C}$. The results obtained are given in Table 1.

Most of the ligands produced active systems in the reaction but with very different activities depending on the substituents at the phosphorus atom. On close inspection, certain trends can be identified. In 
general the order of decreasing activity depending on the heterocycle is $\mathrm{DBF}>>\mathrm{DBT}>\mathrm{DBTO} 2>>\mathrm{TA}$; indeed, the two TA-based systems are completely inactive even at $6 \mathrm{~h}$ reaction time (Entries 3 and 13 ). This order correlates quite nicely with the coordination ability of the heteroatom in the heterocycle so it is not surprising that the two TA-based phosphanes give completely inactive systems given the fact that they act as true bidentate ligands, which are known to inhibit the hydrovinylation reaction.[2c] Within the DBF- and DBT-based families of ligands the order depending on the R substituent is $\mathrm{tBu}>\mathrm{iPr}>\mathrm{Fc}$ $>>\mathrm{OMe} \approx \mathrm{Me}$, which means that, roughly, the bulkier the ligand the more active the system becomes. This trend contrasts with previous results obtained with diarylphosphanes.[15c] It can be observed that except for methoxy-and methylphosphanes (Entries 1-5,10) the change of a polycyclic aryl group $[5,6,15 c, 16]$ for a heterocyclic substituent makes the system much more active but less selective in the hydrovinylation reaction. With regard to the enantioselectivities, they are in the low range but comparable with those obtained with many previously reported diarylphosphanes.[5,6,15c,16] An interesting point is the inversion in the sense of enantioselection in comparison of analogous DBF and DBT phosphanes (cf. Entries 6 and 7 with 10 and 11, respectively) and in each family another inversion in comparison of the systems based on Fcphosphanes (Pd8 and Pd12) with their counterparts (cf. Entries 6,7 with 8,10 and 11 with 12 ).

\section{Pd-Catalysed Allylic Substitution}

209 Asymmetric allylic substitution is a benchmark reaction very often used to test new ligands, especially bidentate ones.[17] In the asymmetric version the model substrate is rac-trans-1,3-diphenylprop-2-enyl acetate (rac-I)[18] and two of the most typically employed nucleophiles are the carbanion derived from dimethyl malonate (DMM, alkylation), formed in situ in the presence of bis(trimethylsilyl)acetamide (BSA) and potassium acetate,[19] and benzylamine (amination), as depicted in Scheme 7. In previous reports, [5,7] we employed Pd complexes of the type $[\operatorname{Pd}\{\eta 3-(2-$ methylallyl)(PArPhR)2\}]PF6 with P-stereogenic diarylphosphanes, obtaining complete conversions at $24 \mathrm{~h}$ and up to $80 \%$ ee in alkylation with a phosphinite ligand at room temperature. Table 2 gives the results obtained with the precursors presented in this paper. In the alkylation reaction, all the cationic and even neutral (Entries 4, 6, 11 and 13) Pd precursors led to full conversion after $24 \mathrm{~h}$, giving the alkylation product with a wide range of enantiopurities depending on the substituents on the phosphorus ligand. It is clear that regardless of the heterocyclic substituent in the ligand those precursors containing phosphinites and methylphosphanes are bad enantioinductors (Entries 1-6 and 12), except for Pd13', which is moderately enantioselective (Entry 16). With regard to the effect of the heterocycle, in general precursors with a DBF-containing ligands are less stereoselective, with the exception of Pd8' (Entry 11). As expected, neutral complexes provide lower but published results.[7,20] The best precursor is Pd11' (Entry 13), the cationic complex with ligand L11. It 
227 is interesting to note that the same ligand was also the most stereoselective in transfer hydrogenation

228 with Ru.[4]

229 The sense of the enantioinduction in the alkylation product also depends on the substituents on the

230 phosphane. It is S for most of the precursors, but the sense is inverted in the case of Fc- and TA-

231 containing precursors, which give rise to the predominant formation of the $\mathrm{R}$ enantiomer of the

232 alkylation product. As expected, neutral complexes provide lower but still moderate levels of

233 stereoselection (cf. Entry 13 with 14 and 16 with 17), in line with previously published results.[7,20]

234 In the case of allylic amination, full conversion is also reached with all the precursors except for cationic

235 complexes Pd3', Pd8', Pd12' and complex Pd6. As expected[5] the enantioselectivities are lower, but

236 follow approximately the same trends as for the allylic alkylation.

237 


\section{CONCLUSIONS}

239

240 In this paper the coordination of 13 optically pure P-stereogenic diaryl monophosphinites and

241 monophosphanes of the type $\mathrm{PPh}(\mathrm{Het}) \mathrm{R}(\mathrm{Het}=4-\mathrm{DBF}, 4-\mathrm{DBT}, 1-\mathrm{TA}$ and 4-DBTO2; $\mathrm{R}=\mathrm{OMe}, \mathrm{Me}$,

$242 \mathrm{iPr}, \mathrm{tBu}, \mathrm{Fc}$ ) to the $\mathrm{Pd}-\eta 3$-methallyl moiety has been studied. It has been found that the only ligands

243 capable of acting as bidentate are those containing a 1-thianthrenyl group. The obtained Pd complexes

244 were used in catalytic asymmetric hydrovinylation of styrene and allylic substitution on rac-I with the

245 aim of comparing the performance of the new ligands with that of previously reported systems based on

$246 \mathrm{P}$-stereogenic $\mathrm{PArPhR}(\mathrm{Ar}=$ polycyclic aromatic group).[5-7,15c,16] It was found that, in general, the

247 new heterocyclic ligands give more active systems for the hydrovinylation reaction but that they are less

248 selective towards 3-phenylbut-1-ene and none of them improves on the best enantioselectivity achieved

249 with the previous systems. For the Pd-catalysed allylic substitution reactions, the activities and the best

250 enantioselectivities (up to $70 \%$ ee) are comparable with those achieved with the published analogous

251 precursors. 
General: All compounds were prepared under purified nitrogen with use of standard Schlenk and vacuum-line techniques. The solvents were purified with a solvent purification system or by standard procedures[21] and kept under nitrogen. $1 \mathrm{H}, 13 \mathrm{C}\{1 \mathrm{H}\}$, and 31P $\{1 \mathrm{H}\}$ and HSQC 1H-13C NMR spectra were recorded with 300 and $400 \mathrm{MHz}$ spectrometers and $\mathrm{CDCl} 3$ as solvent unless otherwise specified. In the NMR spectroscopic data for the Pd-methallyl complexes the following notation has been used: $\mathrm{c}$ (cis) and $t$ (trans) with regard to the phosphorus moiety and s (syn) and a (anti) with regard to the methyl group of the methallyl moiety. IR spectra were recorded in $\mathrm{KBr}$ and the main absorption bands are expressed in $\mathrm{cm}-1$. The results of elemental analyses were not accurate for all compounds, probably due to the presence of residual solvents (as shown in the NMR) or to bad combustion. In these cases, HRMS (carried out with use of electrospray ionisation) clearly reflected the purity of the complexes (see Supporting Information, with assignment of relevant peaks). Styrene hydrovinylation reactions were analysed by $\mathrm{GC}$ with $\mathrm{He}$ as a carrier gas. Allylic substitution reactions on trans-1,3-diphenylprop-2-enyl acetate (rac-I) were analysed by HPLC with a multidiode array detector and a OD-H chiral column $(25 \times$ $0.46 \mathrm{~cm}$ ). The eluent in the analyses was an n-hexane/iPrOH 95:5 mixture for the alkylations and a 99:1 mixture for the aminations. Pd dimer $\mathrm{D}[22]$ and substrate rac-I[23] were prepared by literature procedures whereas other reagents were used as received from commercial suppliers.

\section{Synthesis of the Complexes}

$274[\mathrm{PdCl}(\eta 3-\mathrm{C} 4 \mathrm{H} 7)(\mathrm{L} 1)](\mathrm{Pd} 1)$ : Phosphinite L1 (191 mg, $0.62 \mathrm{mmol})$ was dissolved in dichloromethane $(20 \mathrm{~mL})$, Pd dimer D (102 mg, $0.26 \mathrm{mmol})$ was added, and the yellow solution was stirred for $1 \mathrm{~h}$. The solvent was removed under vacuum and the residue was recrystallized from dichloromethane/hexane, to furnish the title product as a pale yellow solid, yield $150 \mathrm{mg}$ (57\%). 1H NMR (400 MHz): Eur. J. Inorg. Chem. 2016, 4216-4225 www.eurjic.org 4221 (C) 2016 Wiley-VCH Verlag GmbH \& Co. KGaA, Weinheim $\delta=8.07$ (s, $1 \mathrm{H}), 8.06(\mathrm{~s}, 1 \mathrm{H}), 7.97(\mathrm{~s}, 1 \mathrm{H}), 7.95$ (s, $1 \mathrm{H}), 7.91-7.86(\mathrm{~m}, 4 \mathrm{H}), 7.78-7.68$ (m, 2 H), 7.43-7.46 (m, 12 H), 7.38-7.34 (m, 4 H), 4.55 (s, 1 Hts), 4.53 (s, 1 Hts), 3.98 (d, 3JH,P = 14.0 Hz, $3 \mathrm{H}), 3.95(\mathrm{~d}, 3 \mathrm{JH}, \mathrm{P}=12.0 \mathrm{~Hz}, 3 \mathrm{H}), 3.63(\mathrm{~d}, 3 \mathrm{JH}, \mathrm{P}=11.2 \mathrm{~Hz}, 1 \mathrm{Hta}), 3.60$ (d, 3JH,P = 11.6 Hz, $1 \mathrm{Hta})$, 2.93 (s, $1 \mathrm{Hcs}$ ), 2.88 (s, $1 \mathrm{Hcs}), 2.71$ (s, $1 \mathrm{Hca}), 2.70$ (s, $1 \mathrm{Hca}), 1.94$ (s, $3 \mathrm{H}), 1.91$ (s, $3 \mathrm{H})$ ppm. 13C $\{1 \mathrm{H}\}$ NMR (101 MHz): $\delta=155.9-111.8(\mathrm{C}, \mathrm{CH}, \mathrm{Ar}), 79.54$ (d, 2JC,P = 37.3 Hz, CH2t), 79.36 (d, $2 \mathrm{JC}, \mathrm{P}=37.1 \mathrm{~Hz}, \mathrm{CH} 2 \mathrm{t}), 59.2(\mathrm{~s}, \mathrm{CH} 2 \mathrm{c}), 58.7$ (s, CH2c), 57.0 (s, $2 \times \mathrm{CH} 3), 23.2(\mathrm{~s}, 2 \times \mathrm{CH} 3) \mathrm{ppm}$. 31P $\{1 \mathrm{H}\}$ NMR (121 MHz): $\delta=+115.1(\mathrm{~s}),+114.0$ (s) ppm. IR: $v^{\sim}=3052,2935,2835,1583,1482$, 1468, 1435, 1402, 1185, 1108, 1029, 805, 757, $693 \mathrm{~cm}-1$. HRMS: calcd. for C23H22O2PPd [M - Cl] 
[PdCl( $\eta 3-\mathrm{C} 4 \mathrm{H} 7)(\mathrm{L} 2)](\mathrm{Pd} 2)$ : The procedure was the same as that used to prepare Pd1. Starting from L2 (310 $\mathrm{mg}, 0.96 \mathrm{mmol})$ and dimer $\mathrm{D}(134 \mathrm{mg}, 0.34 \mathrm{mmol})$ the desired complex was obtained as a yellowish solid, yield $157 \mathrm{mg}$ (44 \%). 1H NMR (400 MHz): $\delta=8.27$ (t, J = 1.2 Hz, $1 \mathrm{H}$ ), 8.25 (t, J = 1.2 Hz, 1 H), 8.17 (m, 2 H), 7.93-7.80 (m, 4 H), 7.57 (br. m, 1 H), 7.49-7.41 (m, 7 H), 4.60 (s, 1 Hts), 4.58 (s, $1 \mathrm{Hts}), 3.95(\mathrm{~d}, 3 \mathrm{JH}, \mathrm{P}=14.0 \mathrm{~Hz}, 3 \mathrm{H}), 3.91(\mathrm{~d}, 3 \mathrm{JH}, \mathrm{P}=14.0 \mathrm{~Hz}, 3 \mathrm{H}), 3.68$ (d, 3JH,P = $11.2 \mathrm{~Hz}, 2$ Hta), 2.91 (br. s, 1 Hcs), 2.84 (br. s, 1 Hcs), 2.70 (br. s, 1 Hca), 2.65 (br. s, 1 Hca), 1.94 (s, 3 H), 1.91 (s, $3 \mathrm{H})$ ppm. 13C $\{1 \mathrm{H}\}$ NMR (101 MHz): $\delta=134.3-121.6(\mathrm{C}, \mathrm{CH}, \mathrm{Ar}), 80.5(\mathrm{~d}, 2 \mathrm{JC}, \mathrm{P}=18.3 \mathrm{~Hz}, \mathrm{CH} 2 \mathrm{t})$, 80.2 (d, 2JC,P = $19.0 \mathrm{~Hz}, \mathrm{CH} 2 \mathrm{t}$ ), 59.1 (s, CH2 c), 58.8 (s, CH2 c), 56.7 (s, $2 \times \mathrm{CH} 3), 23.34$ (s, CH3), 23.27 (s, CH3) ppm. 31P $\{1 \mathrm{H}\}$ NMR (162 MHz): $\delta=+119.8$ (s), +118.8 (s) ppm. IR: $v^{\sim}=3051,2933$, 2835, 1436, 1376, 1103, 1079, 1028, 805, 754, 693, 585, $555 \mathrm{~cm}-1$. C23H22ClOPPdS (519.31): calcd. C 53.19, H 4.27, S 6.17; found C 52.74, H 4.52, S 5.74.

300

301

$[\mathrm{PdCl}(\eta 3-\mathrm{C} 4 \mathrm{H} 7)(\mathrm{L} 3)](\mathrm{Pd} 3)$ : The procedure was the same as that used to prepare Pd1. Starting from L3 302 (220 mg, $0.62 \mathrm{mmol}$ ) and dimer D (87 mg, $0.22 \mathrm{mmol}$ ) the desired complex was obtained as a pale 303 yellow solid, yield $220 \mathrm{mg}(91 \%)$. 1H NMR (400 MHz): $\delta=7.97$ (dd, J = 7.6, 1.6 Hz, $1 \mathrm{H}), 7.94$ (dd, J 304 = 7.2, $2.0 \mathrm{~Hz}, 1 \mathrm{H}), 7.63-7.56$ (m, $2 \mathrm{H}), 7.47-7.35$ (m, 6 H), 7.24-7.20 (m, $2 \mathrm{H}), 3.88$ (d, 3JH,P = 14.0 305 Hz, $3 \mathrm{H})$ ppm. 31P $\{1 \mathrm{H}\}$ NMR (162 MHz): $\delta=+111.2$ (br. s) ppm. IR: $v^{\sim}=3058,2923,1634,1435$, 306 1382, 1101, 1028, 777, 744, 693, 569, 549, $495 \mathrm{~cm}-1$. C23H22ClOPPdS2 (551.37): calcd. C 50.10, H 4.02, S 11.63; found C 50.40, H 4.21, S 11.88.

$[\mathrm{PdCl}(\eta 3-\mathrm{C} 4 \mathrm{H} 7)(\mathrm{L} 4)](\mathrm{Pd} 4)$ : The procedure was the same as that used to prepare Pd1. Starting from L4

(74 $\mathrm{mg}, 0.21 \mathrm{mmol}$ ) and dimer D (35 mg, $0.088 \mathrm{mmol})$ the desired complex was obtained as a yellow solid, yield $70 \mathrm{mg}$ (72 \%). 1H NMR (400 MHz): $\delta=8.09-8.02$ (m, $4 \mathrm{H}), 7.84-7.78$ (m, $6 \mathrm{H}), 7.67-7.62$ (m, 6 H), 7.58-7.46 (m, 12 H), 4.60 (dd, J = 7.5, 3.2 Hz, 1 Hts), 4.57 (dd, J = 7.5, 3.2 Hz, 1 Hts), 3.93 $(\mathrm{d}, 3 \mathrm{JH}, \mathrm{P}=14.0 \mathrm{~Hz}, 3 \mathrm{H}), 3.92(\mathrm{~d}, 3 \mathrm{JH}, \mathrm{P}=14.0 \mathrm{~Hz}, 3 \mathrm{H}), 3.67$ (d, 3JH,P = 11.2 Hz, $1 \mathrm{Hta}), 3.62$ (d, 3JH,P = 10.8 Hz, $1 \mathrm{Hta}$ ), 3.41 (br. s, $1 \mathrm{Hcs}$ ), 3.33 (br. s, $1 \mathrm{Hcs}$ ), 2.96 (s, $1 \mathrm{Hca}$ ), 2.69 (s, $1 \mathrm{Hca}$ ), 2.01 (s, $3 \mathrm{H}), 1.91(\mathrm{~s}, 3 \mathrm{H}) \mathrm{ppm} .13 \mathrm{C}\{1 \mathrm{H}\} \mathrm{NMR}(101 \mathrm{MHz}): \delta=156.4-121.3(\mathrm{C}, \mathrm{CH}, \mathrm{Ar}), 80.5(\mathrm{~d}, 2 \mathrm{JC}, \mathrm{P}=$ $12.0 \mathrm{~Hz}, \mathrm{CH} 2 \mathrm{t}$ ), 80.2 (d, 2JC,P = 12.7 Hz, CH2 t), 59.1 (d, 2JC,P = 2.3 Hz, CH2 c), 58.5 (d, 2JC,P = 2.8 $\mathrm{Hz}, \mathrm{CH} 2 \mathrm{c}$ ), 57.5 (d, 2JC,P = 2.7 Hz, CH3), 56.9 (d, 2JC,P = 1.9 Hz, CH3), 23.3 (s, $2 \times \mathrm{CH} 3$ ) ppm. 31P $\{1 \mathrm{H}\}$ NMR (162 MHz): $\delta=126.0$ (s), 124.7 (s) ppm. IR: $v^{\sim}=3052,2938,1437,1309,1159,1044$, 764, 584, 568, $543 \mathrm{~cm}-1$. HRMS: calcd. for C23H22O3PPdS [M - Cl] 515.0056; found 515.0079.

[PdCl( $\eta 3-\mathrm{C} 4 \mathrm{H} 7)(\mathrm{L} 5)](\mathrm{Pd} 5)$ : The procedure was the same as that used to prepare Pd1. Starting from L5 (189 $\mathrm{mg}, 0.65 \mathrm{mmol})$ and dimer $\mathrm{D}(106 \mathrm{mg}, 0.27 \mathrm{mmol})$ the desired complex was obtained as a yellow solid, yield $204 \mathrm{mg}$ (78 \%). 1H NMR (400 MHz): $\delta=8.06$ (t, J = 1.2 Hz, $1 \mathrm{H}), 8.04(\mathrm{t}, \mathrm{J}=0.8 \mathrm{~Hz}, 1 \mathrm{H})$, 7.98 (s, 1 H), 7.97-7.96 (m, 2 H), 7.95 (m, 1 H), 7.72-7.64 (m, 4 H), 7.58 (s, 1 H), 7.56 (s, 1 H), $7.51-$ 
Hz, $1 \mathrm{Hta}), 2.94$ (s, $1 \mathrm{Hcs}), 2.87$ (s, $1 \mathrm{Hcs}), 2.66$ (s, $1 \mathrm{Hca}), 2.58$ (s, $1 \mathrm{Hca}$ ), 2.35 (d, 2JH,P = $8.8 \mathrm{~Hz}, 3$ H), $2.33(\mathrm{~d}, 2 \mathrm{JH}, \mathrm{P}=9.2 \mathrm{~Hz}, 3 \mathrm{H}), 1.95(\mathrm{~s}, 3 \mathrm{H}), 1.85(\mathrm{~s}, 3 \mathrm{H}) \mathrm{ppm} .13 \mathrm{C}\{1 \mathrm{H}\} \mathrm{NMR}(101 \mathrm{MHz}): \delta=$ 155.9-111.6 (C, CH, Ar), 76.9 (d, ov, CH2 t), 76.5 (d, ov, CH2 t), 58.9 (s, $2 \times \mathrm{CH} 2$ c), 23.44 (s, CH3), 23.36 (s, CH3), 12.4 (m, $2 \times \mathrm{CH} 3)$ ppm. 31P $\{1 \mathrm{H}\}$ NMR (121 MHz): $\delta=+5.3(\mathrm{~s}),+4.9$ (s) ppm. IR: $v^{\sim}=$ 3050, 2979, 2915, 1583, 1469, 1448, 1435, 1399, 1184, 894, 840, 754, 692, 554, $422 \mathrm{~cm}-1$.

C23H22C1OPPd (487.25): calcd. C 56.69, H 4.55; found C 57.32, H 4.78.

[PdCl( $\eta 3-\mathrm{C} 4 \mathrm{H} 7)(\mathrm{L} 6)](\mathrm{Pd} 6)$ : The procedure was the same as that used to prepare Pd1. Starting from L6 (318 $\mathrm{mg}, 1.00 \mathrm{mmol})$ and dimer D (141 $\mathrm{mg}, 0.36 \mathrm{mmol})$ the desired complex was obtained as a yellow solid, yield $280 \mathrm{mg}$ (75 \%). 1H NMR (400 MHz): $\delta=8.06$ (s, $1 \mathrm{H}), 8.04(\mathrm{~s}, 1 \mathrm{H}), 7.97$ (s, $1 \mathrm{H}), 7.95$ (s, $1 \mathrm{H}), 7.83$ (dd, J = 10.8, 7.6 Hz, $1 \mathrm{H}), 7.75-7.65$ (m, $6 \mathrm{H}), 7.49-7.34$ (m, $13 \mathrm{H}), 4.44$ (dd, J = 6.8, 3.2 Hz, $1 \mathrm{Hts}$ ), 4.42 (dd, J = 6.8, 3.2 Hz, $1 \mathrm{Hts}), 3.53-3.41$ (m, $2 \mathrm{H}), 3.51$ (d, 3JH,P = 9.6 Hz, $1 \mathrm{Hta}), 3.47$ (d, 3JH,P = 10.0 Hz, 1 Hta), 3.05 (m, $1 \mathrm{Hcs}$ ), 2.96 (m, $1 \mathrm{Hcs}$ ), 2.59 (br. s, $2 \mathrm{Hca}$ ), 1.91 (s, $3 \mathrm{H}$ ), 1.77 (s, $3 \mathrm{H}), 1.34-1.19(\mathrm{~m}, 12 \mathrm{H}) \mathrm{ppm} .13 \mathrm{C}\{1 \mathrm{H}\} \mathrm{NMR}(101 \mathrm{MHz}): \delta=156.5-111.7(\mathrm{C}, \mathrm{CH}, \mathrm{Ar}), 77.70(\mathrm{~d}$, 2JC,P = 32.4 Hz, CH2 t), 77.67 (d, 2JC,P = 32.7 Hz, CH2 t), 59.7 (s, CH2 c), 59.0 (s, CH2 c), 24.9 (d, 1JC,P = 18.1 Hz, CH), 24.7 (d, 1JC,P = 18.2 Hz, CH), 23.2 (s, CH3), 23.0 (s, CH3), 19.2 (s, CH3), 19.1 (s, CH3), 18.6 (d, 2JC,P = 1.3 Hz, CH3), 18.4 (s, CH3) ppm. 31P $\{1 \mathrm{H}\}$ NMR (121 MHz): $\delta=+28.0(\mathrm{~s})$, +27.1 (s) ppm. IR: $v^{\sim}=3051,2957,2925,2867,1582,1469,1449,1435,1401,1184,757,697,536$ cm-1. C25H26ClOPPd (515.31): calcd. C 58.27, H 5.09; found C 59.68, H 5.54.

[PdCl( $\eta 3-\mathrm{C} 4 \mathrm{H} 7)(\mathrm{L} 7)](\mathrm{Pd} 7)$ : The procedure was the same as that used to prepare Pd1. Starting from L7 (203 $\mathrm{mg}, 0.61 \mathrm{mmol})$ and dimer D (98 $\mathrm{mg}, 0.25 \mathrm{mmol}$ ) the desired complex was obtained as a yellow solid, yield $150 \mathrm{mg}(57 \%$ ). $1 \mathrm{H}$ NMR (300 MHz): $\delta=8.07-7.86$ (m, $9 \mathrm{H}$ ), 7.78 (ddd, J = 10.5, 7.8, 1.2 Hz, 1 H), 7.49-7.34 (m, 14 H), 4.45-4.40 (m, 2 Hts), 3.53 (d, J = 9.7 Hz, 2 Hta), 2.43 (s, 1 Hcs), 2.25 (s, $1 \mathrm{Hcs}$ ), 2.16 (m, $1 \mathrm{Hca}), 2.12$ (s, $1 \mathrm{Hca}), 1.80$ (s, $3 \mathrm{H}), 1.73$ (s, $3 \mathrm{H}), 1.54$ (d, 3JH,P = 15.8 Hz, $9 \mathrm{H}), 1.50$ $(\mathrm{d}, 3 \mathrm{JH}, \mathrm{P}=15.9 \mathrm{~Hz}, 9 \mathrm{H}) \mathrm{ppm} .13 \mathrm{C}\{1 \mathrm{H}\} \mathrm{NMR}(101 \mathrm{MHz}): \delta=156.1-111.8(\mathrm{C}, \mathrm{CH}, \mathrm{Ar}), 78.3(\mathrm{~d}$, 2JC,P = 31.2 Hz, CH2 t), 78.0 (d, 2JC,P = 31.5 Hz, CH2 t), 61.8 (s, CH2 c), 61.4 (s, CH2 c), 34.9 (d, 1JC,P = 17.7 Hz, C), 34.5 (d, 1JC,P = 17.9 Hz, C), 29.26 (d, 2JC,P = 3.0 Hz, CH3), 29.20 (d, 2JC,P = $3.2 \mathrm{~Hz}, \mathrm{CH} 3), 22.94$ (s, $2 \times \mathrm{CH} 3)$ ppm. 31P $\{1 \mathrm{H}\}$ NMR (121 MHz): $\delta=+35.7$ (s) ppm. IR: $v^{\sim}=3051$, 2956, 2924, 1581, 1469, 1449, 1434, 1398, 1185, 1109, 756, $698 \mathrm{~cm}-1$. C26H28ClOPPd (529.33): calcd. C 58.99, H 5.33; found C 59.42, H 5.78.

[PdCl( $\eta 3-\mathrm{C} 4 \mathrm{H} 7)(\mathrm{L} 8)](\mathrm{Pd} 8)$ : The procedure was the same as that used to prepare Pd1. Starting from L8 (69 $\mathrm{mg}, 0.15 \mathrm{mmol})$ and dimer $\mathrm{D}(25 \mathrm{mg}, 0.064 \mathrm{mmol})$ the desired complex was obtained as a reddish solid, yield $76 \mathrm{mg}$ (90 \%). 1H NMR (400 MHz): $\delta=8.04-8.02$ (m, $2 \mathrm{H}$ ), 7.97 (br. s, $1 \mathrm{H}$ ), 7.95 (br. s, 1 H), 7.75-7.69 (m, 4 H), 7.45-7.30 (m, 14 H), 7.27 (m, 1 H), 4.81 (s, 1 H), 4.70 (s, 1 H), 4.53 (m, 2 Hts), 4.49 (s, $1 \mathrm{H}), 4.47$ (s, $1 \mathrm{H}), 4.44$ (s, $2 \mathrm{H}), 4.35$ (s, $1 \mathrm{H}), 4.28$ (s, $1 \mathrm{H}), 4.243$ (s, 5 H), 4.237 (s, 5 H), 3.65 
(d, 3JH,P = 10.4 Hz, Hta), 3.62 (d, 3JH,P = 10.8 Hz, Hta), 2.90 (s, $1 \mathrm{Hcs}$ ), 2.83 (s, $1 \mathrm{Hca}$ ), 2.76 (s, 1 Hcs), 2.58 (s, $1 \mathrm{Hca}), 1.99$ (s, $3 \mathrm{H}), 1.88(\mathrm{~s}, 3 \mathrm{H})$ ppm. 13C $\{1 \mathrm{H}\}$ NMR $(101 \mathrm{MHz}): \delta=155.7-111.7$ (C, $\mathrm{CH}, \mathrm{Ar}), 78.5$ (d, 2JC,P = 33.6 Hz, CH2 t), 77.9 (d, 2JC,P = 33.6 Hz, CH2 t), 75.3 (d, J = 15.6 Hz, CH), $75.1(\mathrm{~d}, \mathrm{~J}=15.5 \mathrm{~Hz}, \mathrm{CH}), 73.9$ (d, J = 8.9 Hz, CH), 73.8 (d, J = 9.1 Hz, CH), 70.1 (s, 10 CH), 61.6 (s, $\mathrm{CH} 2 \mathrm{c}$ ), 60.1 (s, CH2 c), 23.14 (s, CH3), 23.10 (s, CH3) ppm. 31P $\{1 \mathrm{H}\}$ NMR (162 MHz): $\delta=+6.2$ (s), +6.1 (s) ppm. IR: $v^{\sim}=3087,3050,2958,1618,1469,1448,1403,1265,1184,1166,1108,751,698$ cm-1. HRMS: calcd. for C32H28FeOPPd [M - Cl] 621.0256; found 621.0275.

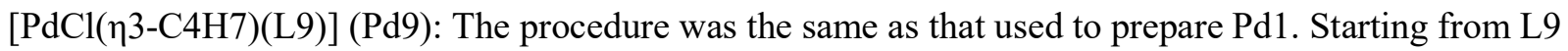
(150 $\mathrm{mg}, 0.33 \mathrm{mmol})$ and dimer D (52 $\mathrm{mg}, 0.13 \mathrm{mmol})$ the desired complex was obtained as a yellow solid, yield $160 \mathrm{mg}$ (93 \%). 1H NMR (400 MHz): $\delta=8.27$ (t, J = 1.6 Hz, $1 \mathrm{H}), 8.25(\mathrm{t}, \mathrm{J}=1.6 \mathrm{~Hz}, 1 \mathrm{H})$, 8.19 (s, 1 H), 8.17 (s, 1 H), 8.11-7.97 (m, 8 H), 7.75-7.02 (m, 2 H), 7.49-7.31 (m, 24 H), 4.55 (m, 2 Hts), 3.69 (d, 3JH,P = 10.0 Hz, $1 \mathrm{Hta}$ ), 3.66 (d, 3JH,P = 10.0 Hz, $1 \mathrm{Hta}$ ), 3.05 (s, $1 \mathrm{Hcs}$ ), 2.86 (s, $1 \mathrm{Hcs}$ ), 2.82 (s, $1 \mathrm{Hca}), 2.70$ (s, $1 \mathrm{Hca}), 2.01$ (s, $3 \mathrm{H}), 1.97$ (s, $3 \mathrm{H})$ ppm. 13C $\{1 \mathrm{H}\} \operatorname{NMR}(101 \mathrm{MHz}): \delta=155.7-$ $111.7(\mathrm{C}, \mathrm{CH}, \mathrm{Ar}), 78.1$ (d, 2JC,P = 32.9 Hz, CH2 t), 77.8 (d, 2JC,P = 32.9 Hz, CH2 t), 62.8 (s, CH2 c), 62.6 (s, CH2 c), 23.06 (s, $2 \times \mathrm{CH} 3)$ ppm. 31P $\{1 \mathrm{H}\}$ NMR (162 MHz): $\delta=+10.4$ (s), +9.3 (s) ppm. IR: v $=3052,1618,1581,1468,1448,1436,1402,1377,1185,1108,800,752,656,541 \mathrm{~cm}-1$. HRMS: calcd. for C34H26OPPdS [M - Cl] 619.0471; found 619.0494. C34H26ClOPPdS (655.47): calcd. C 62.30, S 4.89, H 4.00; found C 61.86, S 4.13, H 4.38.

$[\mathrm{PdCl}(\eta 3-\mathrm{C} 4 \mathrm{H} 7)(\mathrm{L} 10)](\mathrm{Pd} 10)$ : The procedure was the same as that used to prepare Pd1. Starting from L10 (260 mg, $0.85 \mathrm{mmol})$ and dimer D (130 mg, $0.33 \mathrm{mmol})$ the desired complex was obtained as a pale yellow solid, yield $200 \mathrm{mg}$ (60\%). 1H NMR (400 MHz): $\delta=8.24-8.22$ (m, $2 \mathrm{H}), 8.18-8.16(\mathrm{~m}, 2 \mathrm{H})$, 7.79 (br. m, 2 H), 7.72-7.67 (m, 4 H), 7.56-7.43 (m, 14 H), 4.55 (m, 1 Hts), 4.52 (m, 1 Hts), 3.57 (d, 3JH,P = 10.0 Hz, $2 \mathrm{Hta}$ ), 2.95 (s, $2 \mathrm{Hcs}$ ), 2.67 (s, $1 \mathrm{Hca}$ ), 2.55 (s, $1 \mathrm{Hca}), 2.28$ (d, 2JH,P = 8.7 Hz, $3 \mathrm{H}$ ), $2.26(\mathrm{~d}, 2 \mathrm{JH}, \mathrm{P}=8.7 \mathrm{~Hz}, 3 \mathrm{H}), 1.94(\mathrm{~s}, 3 \mathrm{H}), 1.91(\mathrm{~s}, 3 \mathrm{H}) \mathrm{ppm} .13 \mathrm{C}\{1 \mathrm{H}\} \mathrm{NMR}(101 \mathrm{MHz}): \delta=134.6-$ $121.7(\mathrm{C}, \mathrm{CH}, \mathrm{Ar}), 77.8$ (d, 2JC,P = 33.4 Hz, $2 \times \mathrm{CH} 2 \mathrm{t}$ ), 59.1 (s, CH2 c), 58.6 (s, CH2 c), 23.3 (s, $2 \times$ $\mathrm{CH} 3), 12.2(\mathrm{~d}, 1 \mathrm{JC}, \mathrm{P}=27.0 \mathrm{~Hz}, \mathrm{CH} 3), 11.9(\mathrm{~d}, 1 \mathrm{JC}, \mathrm{P}=27.1 \mathrm{~Hz}, \mathrm{CH} 3) \mathrm{ppm} .31 \mathrm{P}\{1 \mathrm{H}\} \mathrm{NMR}(162$ MHz): $\delta=+8.6$ (s), +8.2 (s) ppm. IR: $v^{\sim}=3050,2956,2913,1450,1376,1104,1034,892,754,693$, $520 \mathrm{~cm}-1$. C23H22ClPPdS (503.31): calcd. C 54.88, H 4.41, S 6.37; found C 54.29, H 4.66, S 5.88.

$[\mathrm{PdCl}(\eta 3-\mathrm{C} 4 \mathrm{H} 7)(\mathrm{L} 11)](\mathrm{Pd} 11)$ : The procedure was the same as that used to prepare Pd1. Starting from L11 (461 mg, $1.38 \mathrm{mmol}$ ) and dimer D (181 mg, $0.46 \mathrm{mmol})$ the desired complex was obtained as a yellow solid, yield $255 \mathrm{mg}$ (52 \%). 1H NMR (400 MHz): $\delta=8.26-8.23(\mathrm{~m}, 2 \mathrm{H}), 8.17-8.14(\mathrm{~m}, 2 \mathrm{H})$, $7.85(\mathrm{t}, \mathrm{J}=8.4 \mathrm{~Hz}, 1 \mathrm{H}), 7.78-7.73(\mathrm{~m}, 6 \mathrm{H}), 7.62-7.57$ (m, $3 \mathrm{H}), 7.47-7.35$ (m, $10 \mathrm{H}), 4.50$ (m, $2 \mathrm{Hts})$, $3.60(\mathrm{~d}, 3 \mathrm{JH}, \mathrm{P}=9.2 \mathrm{~Hz}, 1 \mathrm{Hta}), 3.53$ (d, 3JH,P = 9.2 Hz, $1 \mathrm{Hta}), 3.53-3.24$ (m, $2 \mathrm{H}), 2.95$ (br. s, $1 \mathrm{Hcs})$, 2.68 (br. s, $1 \mathrm{Hcs}$ ), 2.64 (s, $1 \mathrm{Hca}$ ), 2.49 (s, $1 \mathrm{Hca}), 1.82$ (s, $6 \mathrm{H}), 1.41$ (dd, J = 6.8, $4.0 \mathrm{~Hz}, 3 \mathrm{H}), 1.36$ 
423

424

425

426

427 (dd, J = 7.2, 4.4 Hz, $3 \mathrm{H}), 1.25$ (dd, J = 16.4, $6.8 \mathrm{~Hz}, 3 \mathrm{H}), 1.18$ (dd, J = 16.4, $6.8 \mathrm{~Hz}, 3 \mathrm{H}) \mathrm{ppm}$. 13C $\{1 \mathrm{H}\}$ NMR (101 MHz): $\delta=139.4-121.6(\mathrm{C}, \mathrm{CH}, \mathrm{Ar}), 79.1(\mathrm{~d}, 2 \mathrm{JC}, \mathrm{P}=31.3 \mathrm{~Hz}, \mathrm{CH} 2 \mathrm{t}), 78.5(\mathrm{~d}$, 2JC,P = 31.0 Hz, CH2 t), 60.3 (s, CH2 c), 58.9 (s, CH2 c), 25.7 (d, 1JC,P = 23.3 Hz, CH), 25.1 (d, 1JC,P $=23.6 \mathrm{~Hz}, \mathrm{CH}), 22.96(\mathrm{~s}, \mathrm{CH} 3), 22.86(\mathrm{~s}, \mathrm{CH} 3), 19.9(\mathrm{~d}, 2 \mathrm{JC}, \mathrm{P}=6.9 \mathrm{~Hz}, \mathrm{CH} 3), 19.8$ (d, 2JC,P = 5.9 Hz, $\mathrm{CH} 3), 18.5(\mathrm{~d}, 2 \mathrm{JC}, \mathrm{P}=2.3 \mathrm{~Hz}, \mathrm{CH} 3), 18.3(\mathrm{~s}, \mathrm{CH} 3) \mathrm{ppm} .31 \mathrm{P}\{1 \mathrm{H}\} \mathrm{NMR}(162 \mathrm{MHz}): \delta=+28.2(\mathrm{~s})$, +28.1 (s) ppm. IR: $v^{\sim}=3048,2958,2925,2866,1435,1374,1097,1079,1034,751,697,659,585,542$, $466 \mathrm{~cm}-1$. HRMS: calcd. for C25H26PPdS [M - Cl] 495.0522; found 495.0542.

$[\mathrm{PdCl}(\eta 3-\mathrm{C} 4 \mathrm{H} 7)(\mathrm{L} 12)](\mathrm{Pd} 12)$ : The procedure was the same as that used to prepare Pd1. Starting from L12 $(60 \mathrm{mg}, 0.13 \mathrm{mmol})$ and dimer D $(21 \mathrm{mg}, 0.053 \mathrm{mmol})$ the desired complex was obtained as a red solid, yield $68 \mathrm{mg}$ (95 \%). 1H NMR (400 MHz): $\delta=8.19-8.13(\mathrm{~m}, 2 \mathrm{H}, \mathrm{M}+\mathrm{m}), 8.10-8.02(\mathrm{~m}, 2 \mathrm{H}, \mathrm{M}$ + m), 7.77-7.73 (m, $1 \mathrm{H}, \mathrm{M}+\mathrm{m}), 7.48-7.41(\mathrm{~m}, 6 \mathrm{H}, \mathrm{M}+\mathrm{m}), 7.22(\mathrm{dd}, \mathrm{J}=10.4,7.6 \mathrm{~Hz}, 1 \mathrm{H}, \mathrm{m}), 7.13$ (dd, J = 10.4, 7.2 Hz, $1 \mathrm{H}, \mathrm{M}), 4.86$ (br. s, $1 \mathrm{H}, \mathrm{m}), 4.75$ (br. s, $1 \mathrm{H}, \mathrm{M}$ ), 4.63 (dd, J = 6.4, 3.2 Hz, $1 \mathrm{Hts}$, M), 4.59 (br. s, 2 H, m), 4.56 (dd, J = 6.8, 3.2 Hz, 1 Hts, m), 4.49-4.47 (m, 2 H, M), 4.29 (s, 5 H, m), 4.28 (s, 5 H, M), 3.98 (br. s, 1 H, m), 3.92 (br. s, 1 H, M), 3.71 (d, 3JH,P = 9.6 Hz, 1 Hta, M), 3.67 (d, 3JH,P = 10.0 Hz, Hta, m), 2.89 (br. s, 1 Hcs, M), 2.80 (br. s, 1 Hcs, m), 2.39 (br. s, 1 Hca, m), 2.15 (br. s, $1 \mathrm{Hca}, \mathrm{M}), 2.07$ (s, $3 \mathrm{H}, \mathrm{M}), 1.62(\mathrm{~s}, 3 \mathrm{H}, \mathrm{m}) \mathrm{ppm} .13 \mathrm{C}\{1 \mathrm{H}\} \mathrm{NMR}(101 \mathrm{MHz}): \delta=134.6-121.6(\mathrm{C}$, $\mathrm{CH}, \mathrm{Ar}), 80.0$ (d, 2JC,P = 32.7 Hz, CH2 t, M), 78.6 (d, 2JC,P = 32.3 Hz, CH2 t, m), 76.1 (s, CH), 75.9 (s, CH), 72.2-71.5 (m, $6 \times \mathrm{CH}), 70.14(\mathrm{~s}, 5 \times \mathrm{CH}, \mathrm{m}), 70.09(\mathrm{~s}, 5 \times \mathrm{CH}, \mathrm{M}), 61.4(\mathrm{~d}, 2 \mathrm{JC}, \mathrm{P}=1.9 \mathrm{~Hz}$, $\mathrm{CH} 2 \mathrm{c}, \mathrm{m}), 59.5$ (s, CH2 c, M), 23.0 (s, CH3, M), 22.9 (s, CH3, m) ppm. 31P $\{1 \mathrm{H}\} \mathrm{NMR}(162 \mathrm{MHz}): \delta$ $=+9.4(\mathrm{~s}, \mathrm{M}),+9.0(\mathrm{~s}, \mathrm{~m}) \mathrm{ppm}$. IR: $v^{\sim}=3048,2958,1437,1378,1272,1167,1108,1097,1078,1023$, 821, 754, 736, 585, 551, 498, 478, 456, $425 \mathrm{~cm}-1$. HRMS: calcd. For C32H28FePPdS [M - Cl] 637.0028 ; found 637.0040 .

$[\mathrm{PdCl}(\eta 3-\mathrm{C} 4 \mathrm{H} 7)(\mathrm{L} 13)](\mathrm{Pd} 13)$ : The procedure was the same as that used to prepare Pd1. Starting from L13 (281 $\mathrm{mg}, 0.83 \mathrm{mmol})$ and dimer D $(130 \mathrm{mg}, 0.33 \mathrm{mmol})$ the desired complex was obtained as a yellow solid, yield $280 \mathrm{mg}$ (79 \%). 1H NMR (400 MHz): $\delta=7.71$ (dd, J = 7.6, 1.6 Hz, $1 \mathrm{H}$ ), 7.68 (dd, J $=7.6,1.6 \mathrm{~Hz}, 1 \mathrm{H}), 7.59(\mathrm{dt}, \mathrm{J}=7.6,1.2 \mathrm{~Hz}, 1 \mathrm{H}), 7.51-7.41(\mathrm{~m}, 4 \mathrm{H}), 7.35(\mathrm{dd}, \mathrm{J}=7.2,1.6 \mathrm{~Hz}, 1$ H), 7.28-7.17 (m, $4 \mathrm{H}), 2.22(\mathrm{~d}, 2 \mathrm{JH}, \mathrm{P}=8.8 \mathrm{~Hz}, 3 \mathrm{H}), 1.91(\mathrm{~s}, 3 \mathrm{H}) \mathrm{ppm} .13 \mathrm{C}\{1 \mathrm{H}\} \mathrm{NMR}(101 \mathrm{MHz}): \delta$ $=139.2-127.2(\mathrm{C}, \mathrm{CH}, \mathrm{Ar}), 23.2(\mathrm{~s}, 2 \times \mathrm{CH} 3), 13.4(\mathrm{~d}, 1 \mathrm{JC}, \mathrm{P}=27.1 \mathrm{~Hz}, \mathrm{CH} 3) \mathrm{ppm} .31 \mathrm{P}\{1 \mathrm{H}\} \mathrm{NMR}$ (162 MHz): $\delta=+8.9$ (br. s) ppm. IR: $v^{\sim}=3048,2956,2911,1616,1448,1434,1380,1140,1101,1027$, 891, 836, 749, 692, 487, 444 cm-1. HRMS: calcd. For C23H22PPdS2 [M - Cl] 498.9929; found 498.9937. C23H22ClPPdS2 (535.37): calcd. C 51.60, S 11.98, H 4.14; found C 50.33, S 11.00, H 4.44.

[Pd( 3-C4H7)(L1)2]PF6 (Pd1'): Phosphinite L1 (323 mg, 1.05 mmol), Pd dimer D (70 mg, 0.18 mmol) and NH4PF6 (171 mg, $1.05 \mathrm{mmol})$ were suspended in dichloromethane $(20 \mathrm{~mL})$ and stirred vigorously for $2 \mathrm{~h}$. Water $(20 \mathrm{~mL})$ was added and the mixture was extracted with dichloromethane $(3 \times 10 \mathrm{~mL})$. 
449

450

451

452

453

454

455

456

457

458

459

460

461

462

463

464

465

466

467

468

469

470

471

472

473

The combined organic phase was washed with water, dried with anhydrous Na2SO4 and filtered, and the solvent was removed under vacuum. The crude product was recrystallised from dichloromethane/hexane, yield $204 \mathrm{mg}$ (62 \%). 1H NMR (400 MHz): $\delta=8.00-7.89$ (m, $4 \mathrm{H}), 7.58-7.20$ (m, 19 H), 7.07 (ddd, J = 10.4, 7.6, 1.2 Hz, $1 \mathrm{H}), 4.06$ (d, J = 5.6 Hz, $1 \mathrm{Hs}), 4.04$ (d, J = 5.6 Hz, $1 \mathrm{Hs}$ ), $3.66(\mathrm{~d}, 3 \mathrm{JH}, \mathrm{P}=13.2 \mathrm{~Hz}, 3 \mathrm{H}), 3.60(\mathrm{~d}, 3 \mathrm{JH}, \mathrm{P}=13.2 \mathrm{~Hz}, 3 \mathrm{H}), 3.47$ (d, 3JH,P = 10.8 Hz, $1 \mathrm{Ha}), 3.39$ (d, $3 \mathrm{JH}, \mathrm{P}=10.8 \mathrm{~Hz}, 1 \mathrm{Ha}), 1.80(\mathrm{~s}, 3 \mathrm{H}) \mathrm{ppm} .13 \mathrm{C}\{1 \mathrm{H}\} \mathrm{NMR}(101 \mathrm{MHz}): \delta=155.6-111.5(\mathrm{C}, \mathrm{CH}, \mathrm{Ar})$, 74.7 (dd, JC,P = 23.0, 3.6 Hz, CH2), 74.4 (dd, JC,P = 25.0, 3.3 Hz, CH2), 56.9 (d, 2JC,P = 6.0 Hz, CH3), $56.8(\mathrm{~d}, 2 \mathrm{JC}, \mathrm{P}=6.6 \mathrm{~Hz}, \mathrm{CH} 3), 23.7(\mathrm{~s}, \mathrm{CH} 3) \mathrm{ppm} .31 \mathrm{P}\{1 \mathrm{H}\} \mathrm{NMR}(162 \mathrm{MHz}): \delta=+113.6(\mathrm{~d}$, 2JP,P $=56.5 \mathrm{~Hz}),+112.4(\mathrm{~d}, 2 \mathrm{JP}, \mathrm{P}=56.5 \mathrm{~Hz}) \mathrm{ppm} . \mathrm{IR}: v^{\sim}=3061,2956,2872,1585,1469,1448,1404$, 1264, 1185, 1110, 1035, 839 [v(PF6 -)], 756, 695, $557 \mathrm{~cm}-1$. C42H37F6O4P3Pd (919.06): calcd. C 54.89, H 4.06; found C 56.52, H 4.98.

[Pd( $13-\mathrm{C} 4 \mathrm{H} 7)(\mathrm{L} 2) 2] \mathrm{PF} 6\left(\mathrm{Pd} 2^{\prime}\right)$ : The procedure was the same as that used to prepare Pd1'. Starting from L2 $(273 \mathrm{mg}, 0.85 \mathrm{mmol})$ and dimer D $(56 \mathrm{mg}, 0.14 \mathrm{mmol})$ the desired complex was obtained as a brown solid, yield $177 \mathrm{mg}$ (66 \%). 1H NMR (400 MHz): $\delta=8.18-8.08$ (m, $4 \mathrm{H}), 7.86(\mathrm{~m}, 1 \mathrm{H}), 7.60-7.30$ (m, 19 H), 4.32 (br. s, 1 Hs), 4.02 (br. s, 1 Hs), 3.52 (d, 3JH,P = 11.6 Hz, 1 Ha), 3.49 (d, 3JH,P = 10.4 Hz, 1 Ha), $3.47(\mathrm{~d}, 3 \mathrm{JH}, \mathrm{P}=12.4 \mathrm{~Hz}, 3 \mathrm{H}), 3.43(\mathrm{~d}, 3 \mathrm{JH}, \mathrm{P}=12.4 \mathrm{~Hz}, 3 \mathrm{H}), 1.87(\mathrm{~s}, 3 \mathrm{H}) \mathrm{ppm} .13 \mathrm{C}\{1 \mathrm{H}\} \mathrm{NMR}$ (101 MHz): $\delta=141.6-121.5(\mathrm{C}, \mathrm{CH}, \mathrm{Ar}), 74.9$ (dd, JC,P = 2.8, $2.7 \mathrm{~Hz}, \mathrm{CH} 2), 74.6$ (dd, JC,P = 2.8, 2.7 $\mathrm{Hz}, \mathrm{CH} 2), 55.8(\mathrm{~d}, 2 \mathrm{JC}, \mathrm{P}=2.1 \mathrm{~Hz}, \mathrm{CH} 3), 55.7(\mathrm{~d}, 2 \mathrm{JC}, \mathrm{P}=2.1 \mathrm{~Hz}, \mathrm{CH} 3), 23.7(\mathrm{~s}, \mathrm{CH} 3) \mathrm{ppm} .31 \mathrm{P}\{1 \mathrm{H}\}$ NMR (162 MHz): $\delta=+122.3(\mathrm{~d}, 2 \mathrm{JP}, \mathrm{P}=55.4 \mathrm{~Hz}),+119.0(\mathrm{~d}, 2 \mathrm{JP}, \mathrm{P}=54.6 \mathrm{~Hz}) \mathrm{ppm}$. IR: $\tilde{v^{\sim}}=3056$, 2940, 1437, 1377, 1104, 1021, 841 [v(PF6 -)], 750, 702, $557 \mathrm{~cm}-1$. HRMS: calcd. For C42H37O2P2PdS2 [M - PF6] 805.0739; found 805.0755.

[Pd( $\eta 3-\mathrm{C} 4 \mathrm{H} 7)(\mathrm{L} 3) 2] \mathrm{PF} 6\left(\mathrm{Pd} 3{ }^{\prime}\right)$ : The procedure was the same as that used to prepare Pd1'. Starting from L3 $(350 \mathrm{mg}, 0.99 \mathrm{mmol})$ and dimer D $(70 \mathrm{mg}, 0.18 \mathrm{mmol})$ the desired complex was obtained as a brown solid, yield $300 \mathrm{mg}$ ( $82 \%$ ). 1H NMR (400 MHz): $\delta=7.62-7.06$ (br. m, $23 \mathrm{H}$ ), 6.78 (br. s, $1 \mathrm{H}$ ), 4.19 (br. s, 2 Hs), 3.54 (br. d, 3JH,P = 9.2 Hz, 3 H), 3.43 (d, 3JH,P = 11.2 Hz, 3 H), 3.33 (br. s, 2 Ha), 2.03 (s, $3 \mathrm{H})$ ppm. 31P $\{1 \mathrm{H}\}$ NMR $(162 \mathrm{MHz}): \delta=+114.5(\mathrm{~d}, 2 \mathrm{JP}, \mathrm{P}=56.2 \mathrm{~Hz}),+112.3(\mathrm{~d}, 2 \mathrm{JP}, \mathrm{P}=56.1 \mathrm{~Hz})$ ppm. IR: $v^{\sim}=3054,2945,2838,1450,1435,1382,1251,1140,1102,1027,832$ [v(PF6 -)], 747, 696, $558 \mathrm{~cm}-1$. C42H37F6O2P3PdS4 (1015.30): calcd. C 49.68, H 3.67, S 12.63; found C 51.89, H 3.03, S 11.76 .

[Pd( $\eta 3-\mathrm{C} 4 \mathrm{H} 7)(\mathrm{L} 5) 2] \mathrm{PF} 6\left(\mathrm{Pd} 5^{\prime}\right)$ : The procedure was the same as that used to prepare Pd1'. Starting from L5 (80 $\mathrm{mg}, 0.28 \mathrm{mmol})$ and dimer $\mathrm{D}(22 \mathrm{mg}, 0.06 \mathrm{mmol})$ the desired complex was obtained as a brownish solid, yield $86 \mathrm{mg}$ (87 \%). 1H NMR (400 MHz): $\delta=7.89-7.79$ (m, $4 \mathrm{H}), 7.51-7.30$ (m, $16 \mathrm{H})$, 7.14-6.98 (m, 3 H), 6.68 (dd, J = 11.6, 7.6 Hz, 1 H), 3.95 (s, $1 \mathrm{Hs}), 3.70$ (s, $1 \mathrm{Hs}), 3.51$ (d, 3JH,P = 10.0 Hz, $1 \mathrm{Ha}), 3.44(\mathrm{~d}, 3 \mathrm{JH}, \mathrm{P}=10.0 \mathrm{~Hz}, 1 \mathrm{Ha}), 2.18$ (d, 2JH,P = 8.4 Hz, $3 \mathrm{H}), 1.99$ (d, 2JH,P = $8.8 \mathrm{~Hz}, 3 \mathrm{H})$, 
$1.85(\mathrm{~s}, 3 \mathrm{H}) \mathrm{ppm} .13 \mathrm{C}\{1 \mathrm{H}\}$ NMR $(101 \mathrm{MHz}): \delta=155.5-111.6(\mathrm{C}, \mathrm{CH}, \mathrm{Ar}), 75.0(\mathrm{~d}, \mathrm{JC}, \mathrm{P}=29.4 \mathrm{~Hz}$, CH2), 74.2 (d, JC,P = 30.6 Hz, CH2), 23.8 (s, CH3), 14.8 (dd, 1JC,P = 28.1, 1.1 Hz, CH3), 13.3 (dd, 1JC,P $=28.0,1.9 \mathrm{~Hz}, \mathrm{CH} 3) \mathrm{ppm} .31 \mathrm{P}\{1 \mathrm{H}\} \mathrm{NMR}(162 \mathrm{MHz}): \delta=+0.2(\mathrm{~d}, 2 \mathrm{JP}, \mathrm{P}=40.5 \mathrm{~Hz}),-0.1(\mathrm{~d}$, 2JP,P $=40.5 \mathrm{~Hz}$ ) ppm. IR: $v^{\sim}=3062,2957,2923,1584,1470,1450,1437,1403,1186,1111,1010,896$, 843 [ $v(\mathrm{PF} 6-)], 754,634,557 \mathrm{~cm}-1$. HRMS: calcd. for C42H37O2P2Pd [M - PF6], 741.1304; found 741.1326.

480

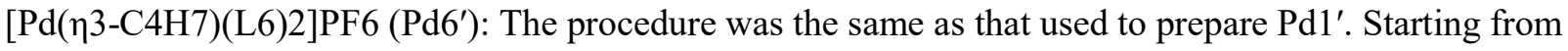
L6 (370 $\mathrm{mg}, 1.16 \mathrm{mmol})$ and dimer D (85 $\mathrm{mg}, 0.22 \mathrm{mmol})$ the desired complex was obtained as a dark yellow solid, yield $290 \mathrm{mg}$ (71 \%). 1H NMR (400 MHz): $\delta=8.01-7.95(\mathrm{~m}, 4 \mathrm{H}), 7.48-7.21(\mathrm{~m}, 18 \mathrm{H})$, 7.16-7.11 (m, 2 H), 4.27 (br. s, $1 \mathrm{Hs}), 4.21$ (br. s, $1 \mathrm{Hs}), 3.42$ (d, 3JH,P = 9.6 Hz, $1 \mathrm{Ha}$ ), 3.31 (d, 3JH,P = $9.6 \mathrm{~Hz}, 1 \mathrm{Ha}), 2.96(\mathrm{~m}, 1 \mathrm{H}), 2.78(\mathrm{~m}, 1 \mathrm{H}), 1.74(\mathrm{~s}, 3 \mathrm{H}), 0.99-0.83(\mathrm{~m}, 12 \mathrm{H}) \mathrm{ppm} .13 \mathrm{C}\{1 \mathrm{H}\} \mathrm{NMR}$ $(101 \mathrm{MHz}): \delta=156.0-111.4(\mathrm{C}, \mathrm{CH}, \mathrm{Ar}), 75.0(\mathrm{~d}, \mathrm{JC}, \mathrm{P}=28.4 \mathrm{~Hz}, \mathrm{CH} 2), 74.7$ (d, JC,P = 28.4 Hz,

CH2), 28.0 (d, 1JC,P = 23.4 Hz, CH), 26.7 (d, 1JC,P = 23.5 Hz, CH), 23.0 (s, CH3), 19.3 (d, 2JC,P = 3.8 $\mathrm{Hz}, \mathrm{CH} 3), 19.1(\mathrm{~d}, 2 \mathrm{JC}, \mathrm{P}=3.6 \mathrm{~Hz}, \mathrm{CH} 3), 19.0(\mathrm{~d}, 2 \mathrm{JC}, \mathrm{P}=2.4 \mathrm{~Hz}, 2 \times \mathrm{CH} 3) \mathrm{ppm} .31 \mathrm{P}\{1 \mathrm{H}\} \mathrm{NMR}(162$ MHz): $\delta=+25.0(\mathrm{~d}, 2 \mathrm{JP}, \mathrm{P}=34.2 \mathrm{~Hz}),+23.7(\mathrm{~d}, 2 \mathrm{JP}, \mathrm{P}=34.2 \mathrm{~Hz}) \mathrm{ppm} . \mathrm{IR}: v^{\sim}=3065,2960,2929$, 2870, 1583, 1469, 1450, 1435, 1402, 1264, 1185, 1111, 1037, 839 [v(PF6 -)], 757, 697, $557 \mathrm{~cm}-1$.

C46H45F6O2P3Pd (943.17): calcd. C 58.58, H 4.81; found C 59.17, H 5.42.

[Pd( $\eta$ 3-C4H7)(L7)2]PF6 (Pd7'): The procedure was the same as that used to prepare Pd1'. Starting from 494 L7 $(190 \mathrm{mg}, 0.57 \mathrm{mmol})$ and dimer $\mathrm{D}(43 \mathrm{mg}, 0.11 \mathrm{mmol})$ the desired complex was obtained as a brown solid, yield $177 \mathrm{mg}$ (83\%). 1H NMR (400 MHz): $\delta=7.79$ (dd, J = 6.0, $3.2 \mathrm{~Hz}, 1 \mathrm{H}), 7.76$ (d, J = 7.2 Hz, $1 \mathrm{H}), 7.68-7.63$ (m, 2 H), 7.58-7.43 (m, 4 H), 7.39-7.14 (m, 11 H), 7.12 (d, J = 8.0 Hz, 1 H), 6.74 (dd, J = 7.2, $4.0 \mathrm{~Hz}, 1 \mathrm{H}), 6.69-6.59(\mathrm{~m}, 2 \mathrm{H}), 6.31(\mathrm{t}, \mathrm{J}=8.8 \mathrm{~Hz}, 1 \mathrm{H}), 5.11(\mathrm{~s}, 1 \mathrm{Hs}), 4.72(\mathrm{~s}, 1 \mathrm{Hs}), 3.75(\mathrm{~d}$, $3 \mathrm{JH}, \mathrm{P}=10.0 \mathrm{~Hz}, 1 \mathrm{Ha}), 3.72(\mathrm{~d}, 3 \mathrm{JH}, \mathrm{P}=10.4 \mathrm{~Hz}, 1 \mathrm{Ha}), 2.35(\mathrm{~s}, 3 \mathrm{H}), 1.18(\mathrm{~d}, 3 \mathrm{JH}, \mathrm{P}=15.6 \mathrm{~Hz}, 9 \mathrm{H})$, $1.03(\mathrm{~d}, 3 \mathrm{JH}, \mathrm{P}=15.2 \mathrm{~Hz}, 9 \mathrm{H}) \mathrm{ppm} .13 \mathrm{C}\{1 \mathrm{H}\} \mathrm{NMR}(101 \mathrm{MHz}): \delta=155.7-111.3(\mathrm{C}, \mathrm{CH}, \mathrm{Ar}), 73.4(\mathrm{~d}$, $\mathrm{JC}, \mathrm{P}=27.5 \mathrm{~Hz}, \mathrm{CH} 2), 72.4$ (d, JC,P = 28.5 Hz, CH2), 36.9 (d, 1JC,P = 20.6 Hz, C), 35.9 (d, 1JC,P = $21.0 \mathrm{~Hz}, \mathrm{C}), 29.8$ (d, 2JC,P = 5.9 Hz, CH3), 29.5 (d, 2JC,P = 6.6 Hz, CH3), 23.0 (s, CH3) ppm. $31 \mathrm{P}\{1 \mathrm{H}\} \operatorname{NMR}(162 \mathrm{MHz}): \delta=+44.4(\mathrm{~d}, 2 \mathrm{JP}, \mathrm{P}=30.5 \mathrm{~Hz}),+43.8(\mathrm{~d}, 2 \mathrm{JP}, \mathrm{P}=30.9 \mathrm{~Hz}) \mathrm{ppm} . \mathrm{IR}: v^{\sim}=$ 3062, 2962, 2869, 1583, 1470, 1449, 1398, 1366, 1264, 1185, 1110, 1094, 1011, 830 [v(PF6 -)], 753, 699, 557 cm-1. C48H49F6O2P3Pd (971.22): calcd. C 59.36, H 5.08; found C 58.90, H 5.52.

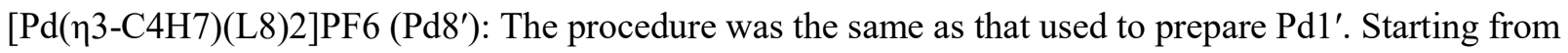
L8 (126 mg, $0.27 \mathrm{mmol})$ and dimer D (21 $\mathrm{mg}, 0.053 \mathrm{mmol})$ the desired complex was obtained as a brown solid, yield $115 \mathrm{mg}(88 \%$ ). 1H NMR (400 MHz): $\delta=8.17$ (d, J = 7.6 Hz, $1 \mathrm{H}), 8.15$ (d, J = 7.2 Hz, 1 H), 7.98 (d, J = 7.6 Hz, 1 H), 7.95 (d, J = 7.2 Hz, 1 H), 7.79 (t, J = 6.4 Hz, 2 H), 7.72-7.68 (m, 3 H), 7.64-7.56 (m, 4 H), 7.41-7.32 (m, 5 H), $7.17(\mathrm{~d}, \mathrm{~J}=8.0 \mathrm{~Hz}, 1 \mathrm{H}), 7.13(\mathrm{~d}, \mathrm{~J}=8.0 \mathrm{~Hz}, 1 \mathrm{H}), 6.54(\mathrm{t}, \mathrm{J}$ 
$=7.6 \mathrm{~Hz}, 1 \mathrm{H}), 6.49(\mathrm{t}, \mathrm{J}=7.6 \mathrm{~Hz}, 1 \mathrm{H}), 6.19(\mathrm{t}, \mathrm{J}=8.8 \mathrm{~Hz}, 1 \mathrm{H}), 6.09(\mathrm{t}, \mathrm{J}=8.8 \mathrm{~Hz}, 1 \mathrm{H}), 4.58(\mathrm{~s}, 1 \mathrm{H})$, $4.47(\mathrm{~s}, 1 \mathrm{H}), 4.40(\mathrm{~s}, 1 \mathrm{H}), 4.38(\mathrm{~s}, 1 \mathrm{H}), 4.31(\mathrm{~s}, 2 \mathrm{H}), 3.96(\mathrm{~s}, 1 \mathrm{H}), 3.93(\mathrm{~s}, 1 \mathrm{Hs}), 3.89$ (s, $1 \mathrm{H}), 3.83$ (s, $1 \mathrm{Hs}), 3.72$ (s, $5 \mathrm{H}), 3.55$ (s, $5 \mathrm{H}), 3.47$ (d, 3JH,P = 10.0 Hz, $1 \mathrm{Ha}), 3.20$ (d, 3JH,P = 10.0 Hz, $1 \mathrm{Ha})$, $1.86(\mathrm{~s}, 3 \mathrm{H}) \mathrm{ppm} .13 \mathrm{C}\{1 \mathrm{H}\} \mathrm{NMR}(101 \mathrm{MHz}): \delta=155.2-111.5(\mathrm{C}, \mathrm{CH}, \mathrm{Ar}), 77.8(\mathrm{~d}, \mathrm{JC}, \mathrm{P}=27.9 \mathrm{~Hz}$, $\mathrm{CH} 2), 74.0(\mathrm{~s}, \mathrm{CH} 2), 73.9-69.7(\mathrm{~m}, 8 \times \mathrm{CH}), 69.5(\mathrm{~s}, 5 \times \mathrm{CH}), 69.3(\mathrm{~s}, 5 \times \mathrm{CH}), 23.4(\mathrm{~s}, \mathrm{CH} 3) \mathrm{ppm}$. $31 \mathrm{P}\{1 \mathrm{H}\}$ NMR $(162 \mathrm{MHz}): \delta=+13.3(\mathrm{~d}, 2 \mathrm{JP}, \mathrm{P}=38.1 \mathrm{~Hz}),+12.9(\mathrm{~d}, 2 \mathrm{JP}, \mathrm{P}=37.7 \mathrm{~Hz}) \mathrm{ppm} . \mathrm{IR}: v^{\sim}=$ 3062, 2956, 1585, 1450, 1437, 1402, 1185, 1162, 1108, 1031, 1002, 839 [v(PF6 -)], 754, 696, $557 \mathrm{~cm}-$ 1. HRMS: calcd. for C60H49Fe2O2P2Pd [M - PF6] 1081.0935; found 1081.0927.

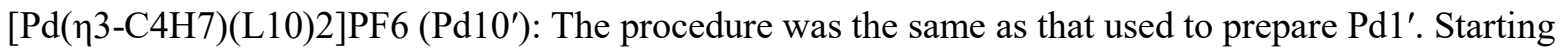
from L10 (370 mg, $1.21 \mathrm{mmol})$ and dimer D (95 mg, $0.24 \mathrm{mmol})$ the desired complex was obtained as a brownish solid, yield $350 \mathrm{mg}$ (79 \%). 1H NMR (400 MHz): $\delta=8.18-8.12(\mathrm{~m}, 4 \mathrm{H}), 7.74-7.72(\mathrm{~m}, 1 \mathrm{H})$, 7.66-7.64 (m, $1 \mathrm{H}), 7.55-7.31$ (m, $13 \mathrm{H}), 7.23$ (td, J = 7.6, 2.4 Hz, $2 \mathrm{H}), 7.17-7.09$ (m, $3 \mathrm{H}), 3.92$ (br. s, $1 \mathrm{Hs}$ ), 3.72 (br. s, $1 \mathrm{Hs}), 3.61$ (d, 3JH,P = 9.6 Hz, $1 \mathrm{Ha}), 3.51$ (d, 3JH,P = 9.6 Hz, $1 \mathrm{Ha}), 2.08$ (d, 2JH,P = $8.4 \mathrm{~Hz}, 3 \mathrm{H}), 1.92(\mathrm{~s}, 3 \mathrm{H}), 1.90(\mathrm{~d}, 2 \mathrm{JH}, \mathrm{P}=8.4 \mathrm{~Hz}, 3 \mathrm{H}) \mathrm{ppm} .13 \mathrm{C}\{1 \mathrm{H}\} \mathrm{NMR}(101 \mathrm{MHz}): \delta=142.6-$ 122.0 (C, CH, Ar), 76.2 (d, JC,P = 28.9 Hz, CH2), 74.9 (d, JC,P = 29.1 Hz, CH2), 23.6 (s, CH3), 13.5 $(\mathrm{d}, 1 \mathrm{JC}, \mathrm{P}=27.9 \mathrm{~Hz}, \mathrm{CH} 3), 12.6(\mathrm{~d}, 1 \mathrm{JC}, \mathrm{P}=27.2 \mathrm{~Hz}, \mathrm{CH} 3) \mathrm{ppm} .31 \mathrm{P}\{1 \mathrm{H}\} \mathrm{NMR}(162 \mathrm{MHz}): \delta=+3.3$ $(\mathrm{d}, 2 \mathrm{JP}, \mathrm{P}=38.2 \mathrm{~Hz}),+1.1(\mathrm{~d}, 2 \mathrm{JP}, \mathrm{P}=38.4 \mathrm{~Hz}) \mathrm{ppm} . \mathrm{IR}: v^{\sim}=3057,1621,1437,1377,1296,1251$, 1161, 1106, 1078, 839 [v(PF6 -)], 753, 693, 557 cm-1. C42H37F6P3PdS2 (919.18): calcd. C 54.88, H 4.06, S 6.98; found C 55.08, H 4.33, S 7.02.

[Pd( $73-\mathrm{C} 4 \mathrm{H} 7)(\mathrm{L} 11) 2] \mathrm{PF} 6\left(\operatorname{Pd1} 1^{\prime}\right)$ : The procedure was the same as that used to prepare Pd1'. Starting from L11 (733 mg, $2.19 \mathrm{mmol})$ and dimer D (166 mg, $0.42 \mathrm{mmol})$ the desired complex was obtained as a brown solid, yield $310 \mathrm{mg}$ (38 \%). 1H NMR (400 MHz): $\delta=8.28$ (t, J = 8.8 Hz, $2 \mathrm{H}), 8.18$ (t, J = 8.4 Hz, 2 H), 7.63-7.44 (m, 10 H), 7.37-7.32 (m, 3 H), 7.24-7.08 (m, 7 H), 4.43 (s, 1 Hs), 4.24 (s, 1 Hs), $3.77(\mathrm{~d}, 3 \mathrm{JH}, \mathrm{P}=9.2 \mathrm{~Hz}, 1 \mathrm{Ha}), 3.53(\mathrm{~d}, 3 \mathrm{JH}, \mathrm{P}=9.6 \mathrm{~Hz}, 1 \mathrm{Ha}), 2.57(\mathrm{~m}, 2 \mathrm{H}), 1.96(\mathrm{~s}, 3 \mathrm{H}), 0.90-0.76$ $(\mathrm{m}, 9 \mathrm{H}), 0.66(\mathrm{dd}, \mathrm{J}=18.0,6.8 \mathrm{~Hz}, 3 \mathrm{H}) \mathrm{ppm} .13 \mathrm{C}\{1 \mathrm{H}\} \mathrm{NMR}(101 \mathrm{MHz}): \delta=143.0-121.9(\mathrm{C}, \mathrm{CH}$, Ar), 76.9 (d, JC,P = 32.0 Hz, CH2), 75.5 (d, JC,P = 28.2 Hz, CH2), 27.1 (d, 1JC,P = 21.2 Hz, CH), 26.6 $(\mathrm{d}, 1 \mathrm{JC}, \mathrm{P}=21.9 \mathrm{~Hz}, \mathrm{CH}), 22.9(\mathrm{~s}, \mathrm{CH} 3), 18.9$ (d, 2JC,P = 4.4 Hz, CH3), $18.5(\mathrm{~s}, 3 \mathrm{CH} 3) \mathrm{ppm} .31 \mathrm{P}\{1 \mathrm{H}\}$ NMR (162 MHz): $\delta=+26.6(\mathrm{~d}, 2 \mathrm{JP}, \mathrm{P}=32.6 \mathrm{~Hz}),+24.9(\mathrm{~d}, 2 \mathrm{JP}, \mathrm{P}=32.6 \mathrm{~Hz}) \mathrm{ppm}$. IR: $v^{\sim}=3057,2962$, 2930, 2870, 1585, 1437, 1375, 1250, 1098, 1078, 1033, 839 [v(PF6-)], 755, 703, $557 \mathrm{~cm}-1$. C46H45F6P3PdS2 (975.29): calcd. C 56.65, H 4.65, S 6.57; found C 56.90, H 5.33, S 6.06.

[Pd( $\eta 3-\mathrm{C} 4 \mathrm{H} 7)(\mathrm{L} 12) 2] \mathrm{PF} 6\left(\mathrm{Pd} 12^{\prime}\right)$ : The procedure was the same as that used to prepare Pd1'. Starting from L12 (100 mg, $0.21 \mathrm{mmol})$ and dimer D $(17 \mathrm{mg}, 0.043 \mathrm{mmol})$ the desired complex was obtained as a brown solid, yield $88 \mathrm{mg}(93 \%$ ). 1H NMR (400 MHz): $\delta=8.03$ (t, J = 6.8 Hz, $2 \mathrm{H}$ ), 7.90 (t, J = 8.8 Hz, 2 H), 7.72 (t, J = 8.4 Hz, 2 H), 7.61 (t, J = 9.2 Hz, 2 H), 7.53-7.34 (m, 12 H), 7.20-6.97 (m, 4 H), 
561

562

563

564

565

566

567

568

569

570

571

572

573

574

575

576

577

578

579

580

581

582

583

584

4.49 (s, $1 \mathrm{H}), 4.40$ (s, $1 \mathrm{H}), 4.35$ (s, $2 \mathrm{H}), 4.30$ (s, $1 \mathrm{H}), 4.23$ (s, $1 \mathrm{Hs}), 4.13$ (s, $1 \mathrm{Hs}), 4.02$ (s, 2 H), 3.96 (s, $1 \mathrm{H}), 3.87(\mathrm{~s}, 5 \mathrm{H}), 3.85(\mathrm{~s}, 5 \mathrm{H}), 3.74(\mathrm{~d}, 3 \mathrm{JH}, \mathrm{P}=12.8 \mathrm{~Hz}, 1 \mathrm{Ha}), 3.71(\mathrm{~d}, 3 \mathrm{JH}, \mathrm{P}=9.6 \mathrm{~Hz}, 1 \mathrm{Ha})$, 2.10 (s, $3 \mathrm{H})$ ppm. 31P $\{1 \mathrm{H}\}$ NMR $(162 \mathrm{MHz}): \delta=+15.0$ (d, 2JP,P = 32.2 Hz), +14.3 (d, 2JP,P = 32.6 Hz) ppm. IR: $v^{\sim}=3057,2957,1437,1402,1376,1161,1107,1033,840[v(\mathrm{PF} 6-)], 754,696,557 \mathrm{~cm}-$ 1. HRMS: calcd. For C60H49Fe2P2PdS2 [M - PF6] 1113.0479; found 1113.0479.

$[\operatorname{Pd}(\eta 3-\mathrm{C} 4 \mathrm{H} 7)(\mathrm{L} 13) 2] \mathrm{PF} 6\left(\operatorname{Pd} 13{ }^{\prime}\right)$ : The procedure was the same as that used to prepare Pd1'. Starting from L13 (240 mg, $0.71 \mathrm{mmol})$ and dimer D (56 mg, $0.14 \mathrm{mmol})$ the desired complex was obtained as a yellow solid, yield $210 \mathrm{mg}$ (76 \%). 1H NMR (400 MHz): $\delta=8.00-6.80$ (m, $24 \mathrm{H}), 3.73$ (br. s, $2 \mathrm{Hs}$ ), 3.49 (br. s, $1 \mathrm{Ha}$ ), 3.25 (br. s, $1 \mathrm{Ha}$ ), 2.03 (s, $3 \mathrm{H}$ ), 2.00 (s, $3 \mathrm{H})$ ppm. 31P $\{1 \mathrm{H}\}$ NMR $(162 \mathrm{MHz}$ ): $\delta=$ +3.4 (d, 2JP,P = 39.7 Hz), +1.5 (d, 2JP,P = 41.1 Hz) ppm. IR: v = 3053, 2957, 2919, 1449, 1434, 1381, 1141, 1110, 1028, 888, 832 [v(PF6 -)], 748, 693, 557 cm-1. HRMS: calcd. for C23H22PPdS2 [M - PF6 - L13] 498.9935; found 498.9950.

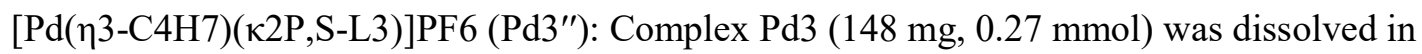
dichloromethane $(20 \mathrm{~mL})$, thallium hexafluorophosphate $(101 \mathrm{mg}, 0.29 \mathrm{mmol})$ was added, and the pale yellow suspension was stirred for $2 \mathrm{~h}$. Water $(20 \mathrm{~mL})$ was added and the mixture was extracted with dichloromethane $(3 \times 10 \mathrm{~mL})$. The combined organic phases were washed with water, dried with anhydrous sodium sulfate and filtered, and the solvent was removed under vacuum. The crude product was recrystallised from dichloromethane/ hexane, yield $120 \mathrm{mg}(67 \%) .1 \mathrm{H} \mathrm{NMR}$ (400 MHz): $\delta=7.89$ 7.87 (d, J = 7.2 Hz, $2 \mathrm{H}, \mathrm{m}+\mathrm{M}), 7.73-7.70(\mathrm{~m}, 5 \mathrm{H}, \mathrm{m}+\mathrm{M}), 7.64-7.41$ (m, $17 \mathrm{H}, \mathrm{m}+\mathrm{M}), 5.36$ (br. s, 1 Hts, m), 5.31 (br. s, 1 Hts, M), 4.64 (s, 1 Hcs, m), 4.31 (s, 1 Hcs, M), 4.20 (d, J = 12.0 Hz, 1 Hta, M), $4.11(\mathrm{~d}, \mathrm{~J}=10.4 \mathrm{~Hz}, 1 \mathrm{Hta}, \mathrm{m}), 3.84(\mathrm{~s}, 1 \mathrm{Hca}, \mathrm{M}), 3.78$ (d, 3JH,P = $15.6 \mathrm{~Hz}, 3 \mathrm{H}, \mathrm{M}), 3.60$ (d, 3JH,P = 15.6 Hz, $3 \mathrm{H}, \mathrm{m}), 3.35$ (s, $1 \mathrm{Hca}, \mathrm{m}), 2.14$ (s, $3 \mathrm{H}, \mathrm{m}), 1.98$ (s, $3 \mathrm{H}, \mathrm{M}) \mathrm{ppm}$. 13C $\{1 \mathrm{H}\}$ NMR $(101$ MHz): $\delta=139.6-129.3$ (C, CH, Ar), 78.9 (d, 2JC,P = 29.8 Hz, CH2 t, m), 78.4 (d, 2JC,P = 32.4 Hz, $\mathrm{CH} 2 \mathrm{t}, \mathrm{M}), 66.1$ (s, CH2 c, M), 65.7 (s, CH2 c, m), 58.1 (s, CH3, M), 57.5 (s, CH3, m), 24.1 (s, CH3, M), 23.9 (s, CH3, m) ppm. 31P $\{1 \mathrm{H}\}$ NMR (162 MHz): $\delta=+138.5$ (br. s, m), 136.2 (s, M) ppm. IR: $v^{\sim}=$ 3058, 2944, 1436, 1386, 1143, 1109, 1026, 832 [v(PF6 -)], 778, 751, 715, 693, 557, $505 \mathrm{~cm}-1$. C23H22F6OP2PdS2 (660.88): calcd. C 41.80, H 3.35, S 9.70; found C 41.87, H 3.61, S 9.99.

$[\mathrm{Pd}(\eta 3-\mathrm{C} 4 \mathrm{H} 7)(\kappa 2 \mathrm{P}, \mathrm{S}-\mathrm{L} 13)] \mathrm{BF} 4\left(\mathrm{Pd13}{ }^{\prime \prime}\right)$ : The procedure was the same as that followed to prepare Pd3". Starting from Pd13 (150 mg, $0.28 \mathrm{mmol})$ and TlBF4 (87 mg, $0.30 \mathrm{mmol})$, the desired complex was obtained as a yellow solid, yield $110 \mathrm{mg}(67 \%) .1 \mathrm{H} \mathrm{NMR}(400 \mathrm{MHz}): \delta=7.83$ (d, J = 7.6 Hz, $1 \mathrm{H}, \mathrm{M}$ ), $7.82(\mathrm{~d}, \mathrm{~J}=7.8 \mathrm{~Hz}, 1 \mathrm{H}, \mathrm{m}), 7.72-7.65(\mathrm{~m}, 5 \mathrm{H}, \mathrm{m}+\mathrm{M}), 7.64-7.43$ (m, $17 \mathrm{H}, \mathrm{m}+\mathrm{M}), 5.28$ (br. s, $1 \mathrm{Hts}$, m), 5.21 (br. s, 1 Hts, M), 4.75 (s, 1 Hcs, m), 4.26 (s, 1 Hcs, M), 4.05 (d, J = 10.0 Hz, 1 Hta, M), 3.88 (d, $\mathrm{J}=10.0 \mathrm{~Hz}, 1 \mathrm{Hta}, \mathrm{m}), 3.77$ (s, $1 \mathrm{Hca}, \mathrm{M}), 3.20$ (s, $1 \mathrm{Hca}, \mathrm{m}), 2.35$ (d, 2JH,P = 10.0 Hz, $3 \mathrm{H}, \mathrm{M}$ ), 2.30 (d, 2JH,P = 10.0 Hz, $3 \mathrm{H}, \mathrm{m}), 2.14(\mathrm{~s}, 3 \mathrm{H}, \mathrm{m}), 1.96$ (s, $3 \mathrm{H}, \mathrm{M}) \mathrm{ppm} .13 \mathrm{C}\{1 \mathrm{H}\} \mathrm{NMR}(101 \mathrm{MHz}): \delta=$ 
604

605

606

607

608

609

610

611

612

139.8-129.5 (C, CH, Ar), 77.0 (d, 2JC,P = 31.7 Hz, CH2 t, m), 76.7 (d, 2JC,P = 31.3 Hz, CH2 t, M), 67.0 (s, CH2 c, M), 66.4 (s, CH2 c, m), 24.0 (s, CH3, M), 23.8 (s, CH3, m), 14.4 (d, 1JC,P = 28.7 Hz, $\mathrm{CH} 3, \mathrm{~m}), 12.9(\mathrm{~d}, 1 \mathrm{JC}, \mathrm{P}=28.3 \mathrm{~Hz}, \mathrm{CH} 3, \mathrm{M}) \mathrm{ppm} .31 \mathrm{P}\{1 \mathrm{H}\} \mathrm{NMR}(162 \mathrm{MHz}): \delta=+35.4(\mathrm{~s}, \mathrm{M}), 34.2$ (s, m) ppm. IR: $v^{\sim}=3056,2958,2918,1436,1385,1283,1046$ [v(BF4-)], 894, 838, 789, 751, 714, 693, 556, 534, 521, 499, 462, 435 cm-1. C23H22BF4PPdS2 (586.72): calcd. C 47.08, H 3.78, S 10.93; found C 46.89, H 4.05, S 10.41.

\section{Catalytic Procedures}

Pd-Catalysed Hydrovinylation: Hydrovinylation reactions were carried out in a stainless steel autoclave fitted with an external jacket connected to an ethanol bath, with the temperature controlled by thermostat to $\pm 0.5^{\circ} \mathrm{C}$. The internal temperature was monitored with a thermocouple. The Pd precursor $(0.020$ $\mathrm{mmol}$ ), styrene (2.08 g, $20 \mathrm{mmol})$ and AgBF4 (4.3 mg, $0.022 \mathrm{mmol})$ were dissolved in dichloromethane $(15 \mathrm{~mL})$ and stirred for $10 \mathrm{~min}$, protected from light. After the $\mathrm{AgCl}$ produced had been filtered off, the solution was quickly placed, by syringe, into the autoclave, which had been purged by successive vacuum/nitrogen cycles and was thermostatted to $25^{\circ} \mathrm{C}$. Ethylene was admitted until a pressure of around 15 bar was reached. After the allotted time, the autoclave was slowly depressurized and aqueous $\mathrm{NH} 4 \mathrm{Cl}$ solution $(10 \%, 10 \mathrm{~mL})$ was added. The mixture was stirred for $10 \mathrm{~min}$ in order to quench the catalyst. The organic layer was separated, dried with $\mathrm{Na} 2 \mathrm{SO} 4$, filtered through a plug of $\mathrm{SiO} 2$ and subjected to GC analysis.

\section{Pd-Catalysed Allylic Substitutions}

(A) Allylic Alkylation with Dimethyl Malonate: The appropriate Pd precursor (0.01 mmol), trans-1,3diphenylprop-2-enyl acetate (rac-I, $1 \mathrm{mmol})$, dimethyl malonate ( $3 \mathrm{mmol})$, BSA ( $3 \mathrm{mmol})$ and KOAc (1 $\mathrm{mg}$ ) were dissolved, in that precise order, in dichloromethane $(5 \mathrm{~mL})$ under nitrogen. The flask was covered with an aluminium foil and left stirring for the allotted time. To quench the reaction, diethyl ether $(20 \mathrm{~mL})$ and aqueous ammonium chloride solution $(10 \%, 20 \mathrm{~mL})$ were added. After extraction, the organic phase was dried with anhydrous sodium sulfate and filtered, and the solvents were removed in vacuo. The crude product was analysed by $1 \mathrm{H}$ NMR to estimate the level of conversion. It was then dissolved in ethyl acetate and passed through a column of silica to remove the metallic impurities. The eluent was removed in vacuo and the residue was analysed by NMR and HPLC.

(B) Allylic Amination with Benzylamine: The Pd precursor (0.01 mmol), trans-1,3-diphenylprop-2-enyl acetate (rac-I, $1 \mathrm{mmol}$ ) and benzylamine $(3 \mathrm{mmol})$ were dissolved, in that precise order, in dichloromethane $(5 \mathrm{~mL})$ under nitrogen. The flask was covered with an aluminium foil and the mixture was stirred for the allotted time. To quench the reaction, diethyl ether $(20 \mathrm{~mL})$ and aqueous ammonium chloride solution $(10 \%, 20 \mathrm{~mL})$ were added. After extraction, the organic phase was dried with anhydrous sodium sulfate and filtered, and the solvents were removed in vacuo. The crude product was analysed by $1 \mathrm{H}$ NMR to estimate the level of conversion. It was then dissolved in ethyl acetate and 
622 passed through a column of silica to remove the metallic impurities. The eluent was removed in vacuo 623 and the residue was analysed by NMR and HPLC.

624

625

626

627

628 


\section{ACKNOWLEDGMENTS}

630

631 The authors thank the Ministerio de Economía y Competitividad (MEC) and the Fondo Europeo de 632 Desarrollo Regional (FEDER) (grant number CTQ2015-65040-P) for financial support of this work. 
634 Keywords: Asymmetric catalysis $\cdot$ Hydrovinylation $\cdot$ Allylic substitution $\cdot$ Palladium $\cdot$ P ligands $\cdot$ 635 Phosphane ligands 
[1] a) T. V. RajanBabu, Chem. Rev. 2003, 103, 2845-2860; b) T. V. RajanBabu, Synlett 2009, 853-885; c) T. V. RajanBabu, C. R. Smith, in: Comprehensive Chirality (Eds.: E. M. Carreira, Y. Hisashi), Elsevier, Amsterdam, 2012, p. 355-398; d) R. M. Ceder, A. Grabulosa, G. Muller, M. Rocamora, Catal. Sci. Technol. 2013, 3, 1446-1464.

[2] a) N. Nomura, J. Jin, H. Park, T. V. RajanBabu, J. Am. Chem. Soc. 1998, 120, 459-460; b) M. Nandi, J. Jin, T. V. RajanBabu, J. Am. Chem. Soc. 1999, 121, 9899-9900; c) T. V. RajanBabu, N. Nomura, J. Jin, M. Nandi, H. Park, X. Sun, J. Org. Chem. 2003, 68, 8431-8446; d) B. Saha, T. V. RajanBabu, J. Org. Chem. 2007, 72, 2357-2363; e) S. Biswas, A. Zhang, B. Raya, T. V. RajanBabu, Adv. Synth. Catal. 2014, 356, 2281-2292.

a) M. Hölscher, G. Franciò, W. Leitner, Organometallics 2004, 23, 5606-5617; b) N.

P. Clavero, A. Grabulosa, M. Rocamora, G. Muller, M. Font-Bardia, Dalton Trans. 2016, 45, $8513-8531$.

P. Clavero, A. Grabulosa, M. Font-Bardia, G. Muller, J. Mol. Catal. A 2014, 391, 183-190.

6] Arabulosa, G. Muller, J. I. Ordinas, A. Mezzetti, M. A. Maestro, M. Font-Bardia, X. Solans, Organometallics 2005, 24, 4961-4973.

[7] A. Grabulosa, G. Muller, R. Ceder, M. A. Maestro, Eur. J. Inorg. Chem. 2010, 3372-3383.

[8] a) D. Alberti, R. Goddard, K.-R. Pörschke, Organometallics 2005, 24, 3907-3915; b) R. M. Ceder, C. Garcia, A. Grabulosa, F. Karipcin, G. Muller, M. Rocamora, M. Font-Bardia, X. Solans, J. Organomet. Chem. 2007, 692, 4005-4019.

[9] P. E. Garrou, Chem. Rev. 1981, 81, 229-266.

[10] a) J. M. Lovell, R. L. Beddoes, J. A. Joule, Tetrahedron 1996, 52, 4745-4756; b) S. Ogawa, H. Muraoka, R. Sato, Tetrahedron Lett. 2006, 47, 2479-2483; c) A. Swist, J. Sloducho, P. Data, M. Lapkowski, ARKIVOC (Gainesville, FL, U.S.) 2012, 193-209; d) M. C. Sheikh, T. Iwasawa, K. Nakajima, A. Kitao, N. Tsubaki, R. Miyatake, T. Yoshimura, H. Morita, Synthesis 2014, 46, $42-48$.

[11] I. H. Filip, E. Gál, I. Lupan, M. Perde Schrepler, P. Lönnecke, M. Surducan, L. Gaina, E. HeyHawkins, L. Silaghi-Dumitrescu, Dalton Trans. 2015, 44, 615-629.

[12] T. V. RajanBabu, N. Nomura, J. Jin, B. Radetich, H. Park, M. Nandi, Chem. Eur. J. 1999, 5, 1963-1968.

[13] G. Franciò, F. Faraone, W. Leitner, J. Am. Chem. Soc. 2002, 124, 736-737.

[14] a) R. Bayersdörfer, B. Ganter, U. Englert, W. Keim, D. Vogt, J. Organomet. Chem. 1998, 552, 187-194; b) R. M. B. Carrilho, G. N. Costa, Â. C. B. Neves, M. M. Pereira, A. Grabulosa, J. C. Bayón, M. Rocamora, G. Muller, Eur. J. Inorg. Chem. 2014, 1034-1041.

[15] a) J. Albert, J. M. Cadena, J. R. Granell, G. Muller, J. I. Ordinas, D. Panyella, C. Puerta, C. Sañudo, P. Valerga, Organometallics 1999, 18, 3511-3518; b) I. Ayora, R. M. Ceder, M. Espinel, G. Muller, M. Rocamora, M. Serrano, Organometallics 2011, 30, 115-128; c) A. 
Grabulosa, A. Mannu, G. Muller, T. Calvet, M. Font-Bardia, J. Organomet. Chem. 2011, 696, $2338-2345$.

677 [16] L. Rodríguez, O. Rossell, M. Seco, A. Grabulosa, G. Muller, M. Rocamora, Organometallics 2006, 25, 1368-1376.

679

[17] a) B. M. Trost, D. L. van Vranken, Chem. Rev. 1996, 96, 395-422; b) B. M. Trost, M. L. Crawley, Chem. Rev. 2003, 103, 2921-2943; c) B. Sundararaju, M. Achard, C. Bruneau, Chem. Soc. Rev. 2012, 41, 4467-4483; d) N. A. Butt, W. Zhang, Chem. Soc. Rev. 2015, 44, $7929-$ 7967.

[18] M. Ramillien, N. Vanthuyne, M. Jean, D. Gherase, M. Giorgi, J.-V. r. Naubron, P. Piras, C. Roussel, J. Chromatogr. A 2012, 1269, 82-93.

[19] H. Aït-Haddou, O. Hoarau, D. Cramailére, F. Pezet, J. Daran, G. G. A. Balavoine, Chem. Eur. J. 2004, 10, 699-707.

687 [20] Y.-Y. Yan, T. V. RajanBabu, Org. Lett. 2000, 2, 199-202.

688 [21] W. L. F. Armarego, C. L. L. Chai, Purification of Laboratory Chemicals, $7^{\text {th }}$ ed., Butterworth 689 Heinemann, Oxford, UK, 2013.

690 [22] W. T. Dent, R. Long, A. J. Wilkinson, J. Chem. Soc. 1964, 1585-1588.

691 [23] P. R. Auburn, P. B. Mackenzie, B. Bosnich, J. Am. Chem. Soc. 1985, 107, 2033-2046.

692 
695

696

697

698

699

700

701

702

703

704

705

706

707

708

709

710

711

712

713

714

715

716

717

718

719

Scheme 1. P-stereogenic phosphanes containing a heterocyclic substituent.

Scheme 2. Preparation of neutral palladium complexes Pd1-13.

Scheme 3. Preparation of cationic Pd complexes Pd1'-13'.

Scheme 4. Unsuccessful attempts to force $\kappa 2 \mathrm{P}, \mathrm{O}-$ and $\kappa 2 \mathrm{P}, \mathrm{S}-$ coordination in Pd complexes of DBF- and DBT-based ligands.

Scheme 5. Successful к2P,S-coordination in Pd complexes of TA-based ligands.

Figure 1. ORTEP representation (thermal ellipsoids drawn at $50 \%$ probability level, $\mathrm{H}$ atoms and PF6 anions removed for clarity) of anti-Pd3" (left) and syn-Pd3" (right). Interatomic distances $[\AA]$ and angles [ ${ }^{\circ}$ f for anti-Pd3": $\mathrm{Pd}(1 \mathrm{~A})-\mathrm{C}(19 \mathrm{~A}), 2.107(11)$; $\mathrm{Pd}(1 \mathrm{~A})-\mathrm{C}(20 \mathrm{~A}), 2.212(10)$; $\mathrm{Pd}(1 \mathrm{~A})-\mathrm{C}(21 \mathrm{~A}), 2.211(10)$; P(1A)-Pd(1A)-C(19A), 98.9(3); C(19A)-Pd(1A)-C(21A), 65.9(4); C(21A)-Pd(1A)-S(1A), 106.7(3); S(1A)-Pd(1A)-P(1A), 88.18(9); C(1A)-S(1A)-C(12A), 100.3(4); C(6A)-S(2A)-C(7A), 100.9(5). For syn-Pd3": Pd(1B)-C(19B), 2.238(10); Pd(1B)-C(20B), 2.167(9); Pd(1B)-C(21B), 2.210(11); P(1B)Pd(1B)-C(21B), 98.8(3); C(21B)-Pd(1B)-C(19B), 66.8(4); C(19B)-Pd(1B)-S(1B), 105.2(3); S(1B)Pd(1B)-P(1B), 88.65(8); C(1B)-S(1B)-C(12B), 100.4(5); C(6B)-S(2B)-C(7B), 102.0(4).

Scheme 6 Pd-catalysed enantioselective hydrovinylation of styrene.

Scheme 7. Pd-catalysed enantioselective allylic substitution on substrate rac-I. 
<smiles>COP(c1ccccc1)(c1ccccc1)(c1ccccc1)c1ccccc1</smiles>

L1

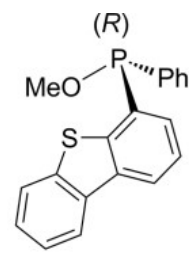

L2

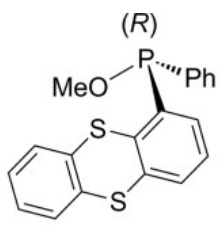

L3

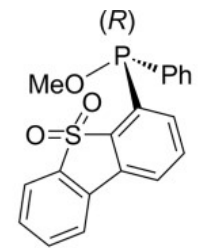

L4

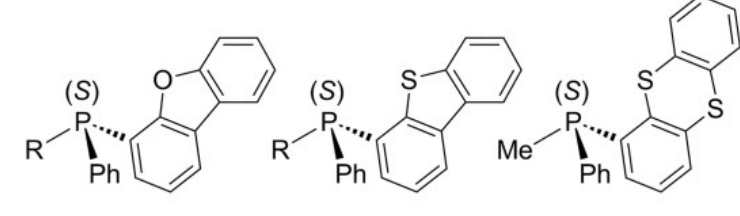

L5-9

$\mathrm{R}=\mathrm{Me}$ (L5)

$\mathrm{R}=i \operatorname{Pr}(\mathbf{L 6})$

$\mathrm{R}=t \mathrm{Bu}(\mathbf{L} 7)$

$\mathrm{R}=\mathrm{Fc}$ (L8)

$\mathrm{R}=\mathrm{DBT}(\mathbf{L} \mathbf{9})$ 
SCHEME 2

726

727

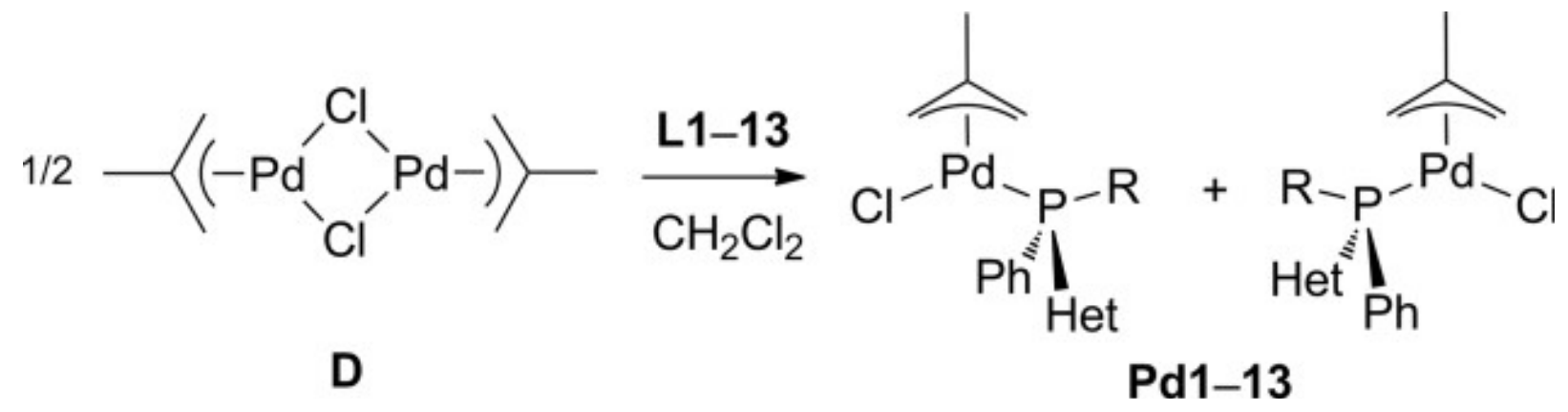


SCHEME 3

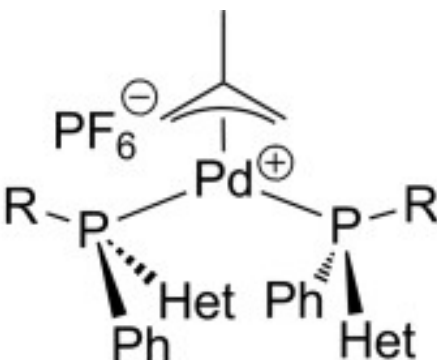




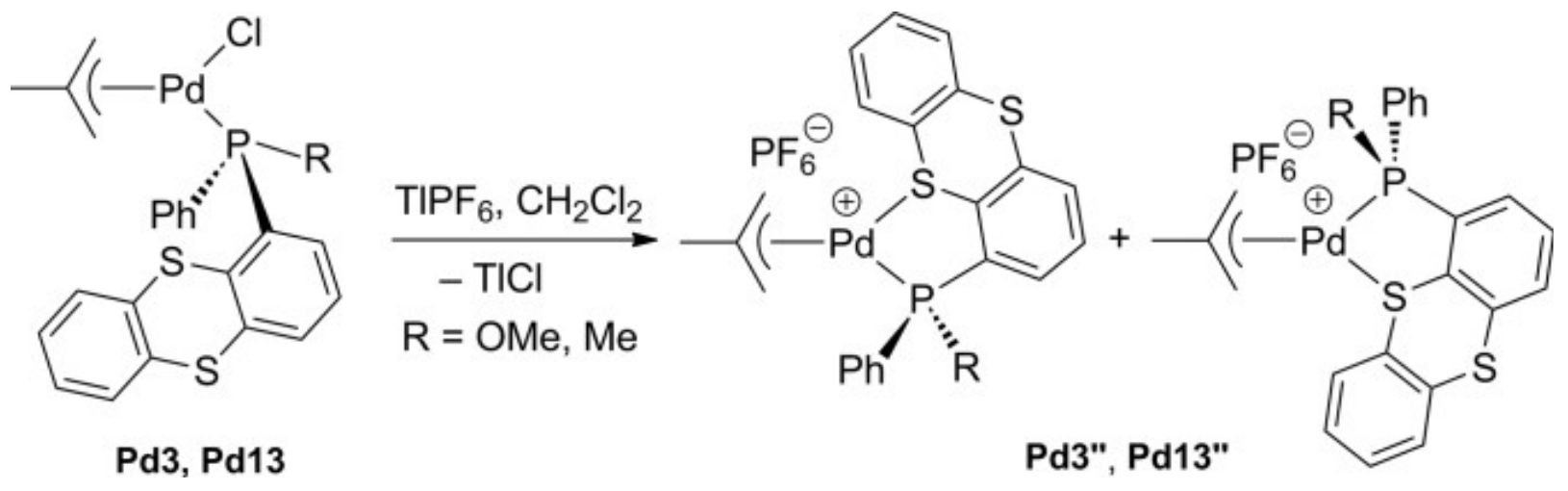


FIGURE 1

751

752

753
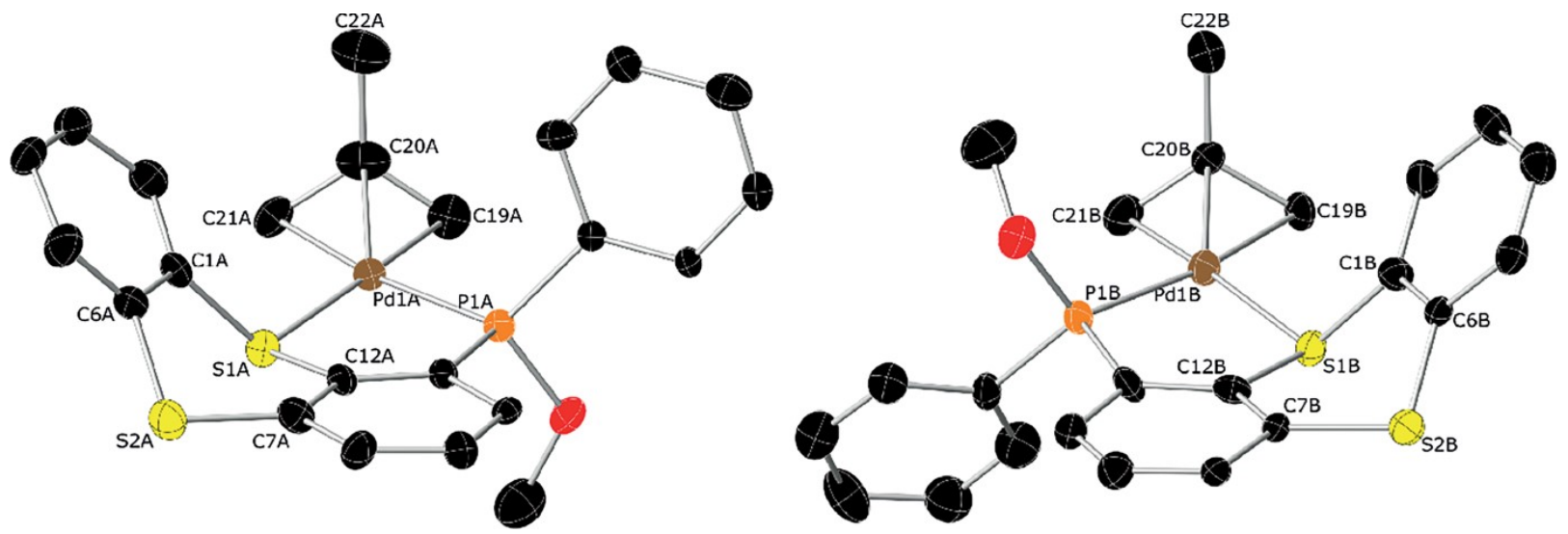
<smiles>C=Cc1ccccc1</smiles>

styrene<smiles>C=C[C@H](C)C1=CCC=C1</smiles><smiles>CC=C(C)c1ccccc1</smiles> 
<smiles>NCc1ccccc1</smiles>

$100 \%$ conversion<smiles>CC(=O)O[C@H](/C=C/c1ccccc1)c1ccccc1</smiles>
rac-I

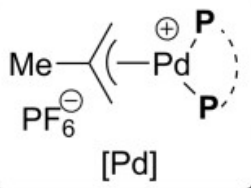<smiles>COC(=O)CC(=O)OC</smiles>

$100 \%$ conversion 
Table 1 Results of styrene hydrovinylation with Pd1-13 complexes.

\begin{tabular}{|c|c|c|c|c|c|c|c|}
\hline Entry & Precursor & Trme [h] & Conversion ${ }^{[\mathrm{BS}}[9 /]$ & Codimers ${ }^{k 1}[\%]$ & Selectivity ${ }^{3 / 1}[6]$ & TOFlo] $\left[\mathrm{h}^{-1}\right]$ & $\mathrm{e}^{n / 2}[\%]$ \\
\hline 1 & Pd1 & 2 & 13.3 & 13.3 & 299 & 66 & $6(9)$ \\
\hline 2 & Pd2 & 4 & 33.2 & 32.9 & 92.6 & 81 & $<5$ \\
\hline 3 & Pd3 & 6 & $<5$ & - & - & - & - \\
\hline 4 & Pd4 & 4 & 18.8 & 18.5 & $>99$ & 50 & $<5$ \\
\hline 5 & Pd5 & 6 & 55.6 & 55.6 & 73.2 & 91 & $<5$ \\
\hline 6 & Pd6 & 0.5 & 58.6 & 57.6 & 92.6 & 1129 & $6(R)$ \\
\hline 7 & Pd7 & 0.5 & 91.4 & 91.4 & 74.9 & 1839 & $13(\mathbb{R})$ \\
\hline 8 & PdB & 1 & 673 & 673 & 80.4 & 667 & 20 (9) \\
\hline 9 & Pd9 & 1 & 48.0 & 48.0 & 91.4 & 476 & $<5$ \\
\hline 10 & Pd10 & 2 & 19.9 & 19.4 & 94.5 & 95 & 10 (9) \\
\hline 11 & Pd11 & 1 & 84.5 & 83.9 & 85 & 822 & 18 [S] \\
\hline 12 & Pd12 & 1 & 67.0 & 66.9 & 85.8 & 659 & $14(\mathbb{R})$ \\
\hline 13 & Pd13 & 6 & $<5$ & - & - & - & - \\
\hline
\end{tabular}

[a] Catalytic conditions: Pd complex $\left(0.02\right.$ mmol, styrene $\left(20 \mathrm{mmol}, \mathrm{AgBF}_{4}, 0.022 \mathrm{mmol}\right)$ at $25 \tau$ and $\mathrm{P}=15$ bar of initial pressure of ethylene in $15 \mathrm{~mL}$ of dichloromethane [b] Conversion of starting styrene [c] Total amount of codimers. [d] Percentsge of 3-phernlbut-1-ene/codimers [e] TOF values calaulated from the total amount of codimers formed. [f] Enantiomeric ercess of 3-phenylbut-1-ene at the stated time. 
774 Table 2. Results of enantioselective allylic substitutions of rac-I with Pd complexes.

\begin{tabular}{|c|c|c|c|c|}
\hline Entry $^{|2|}$ & Nucleophile & Precarsor & Corwersion $[\%]^{|b|}$ & $\approx[\$ 6]^{k 1}$ \\
\hline 1 & DMM & Pd1' & 299 & es \\
\hline 2 & DMM & $\mathrm{Pd} 2$ & $>99$ & 19 [9] \\
\hline 3 & DMM & Pd2' & $>99$ & $6(R)$ \\
\hline 4 & DMM & Pd3 & $>99$ & $6(5)$ \\
\hline 5 & DMM & $\mathrm{Pd}^{\prime \prime}$ & 299 & $8(5)$ \\
\hline 6 & DMM & PdS' & 299 & $<5$ \\
\hline 7 & DMM & Pd6' & 299 & 12 (ग) \\
\hline 8 & DMM & Pd6 & 299 & $16[9]$ \\
\hline 9 & DMM & $\mathrm{Pd} 7^{\prime}$ & 299 & $6(9)$ \\
\hline 10 & DMM & Pd7 & 299 & $<5$ \\
\hline 11 & DMM & Pd8 & 299 & $51(R)$ \\
\hline 12 & DMM & Pd10' & $>99$ & $<5$ \\
\hline 13 & DMM & Pd11' & 299 & 70 [9] \\
\hline 14 & DMM & Pd11 & 299 & $58[9]$ \\
\hline 15 & DMM & Pd12' & 299 & $40(n)$ \\
\hline 16 & DMM & Pd13' & $>99$ & $43(R)$ \\
\hline 17 & DMM & Pd13 & 299 & $34(R)$ \\
\hline 18 & DMM & Pd13" & 299 & $23(R)$ \\
\hline 19 & benxylamine & Pd1' & 299 & $<5$ \\
\hline 20 & benxylamine & $\mathrm{Pd} 2$ & 299 & $<5$ \\
\hline 21 & benxylamine & $\mathrm{Pd}^{\prime}$ & 95 & $18[9]$ \\
\hline 22 & benxylamine & $\mathrm{Pd} 3^{*}$ & 299 & $9(9)$ \\
\hline 23 & benxylamine & PdS' & $>99$ & $<5$ \\
\hline 24 & benxylamine & Pd6' & 299 & $<5$ \\
\hline 25 & benxylamine & Pd6 & 31 & $<5$ \\
\hline 26 & benxylamine & $\mathrm{Pd} 7^{\prime}$ & $>99$ & $9[9]$ \\
\hline 27 & benxylamine & Pd7 & 299 & $<5$ \\
\hline 28 & benxylamine & Pd8 & 88 & $53(9)$ \\
\hline 29 & benxylamine & Pd10' & $>99$ & $<5$ \\
\hline 30 & benxylamine & Pd11' & 299 & $45(R)$ \\
\hline 31 & benxylamine & Pd12' & 50 & $13(9)$ \\
\hline 32 & benxylamine & Pd13' & 299 & $38[9]$ \\
\hline 33 & benxylamine & Pd13 & 299 & $49[9]$ \\
\hline 34 & benxylamine & Pd13" & 299 & 33 (9) \\
\hline
\end{tabular}

a) Catalytic conditions for allylic alkylations with DMML Pd complex (0.01 mmol), ract (1 menol, dimethyl malonate [3 mmol), BSA [3 mmol) and KOAc (1 mq) in $5 \mathrm{~mL}$ of $\mathrm{CH}_{2} \mathrm{O}_{2}$ at room ternp. for $24 \mathrm{~h}$, for alhylic substitutions with benxylamine Pd complex (0.01 mmol), ract (1 mmol) and benzylamine (3 mmol) in $5 \mathrm{~mL}$ of $\mathrm{CH}_{2} \mathrm{Cl}_{2}$ at room temp. for $24 \mathrm{~h}$. [b] Percentage corversion expressed as rac-l consumption, determined by NMR and HPLC. [c] Ensntiomeric excesses determined by HPLC 